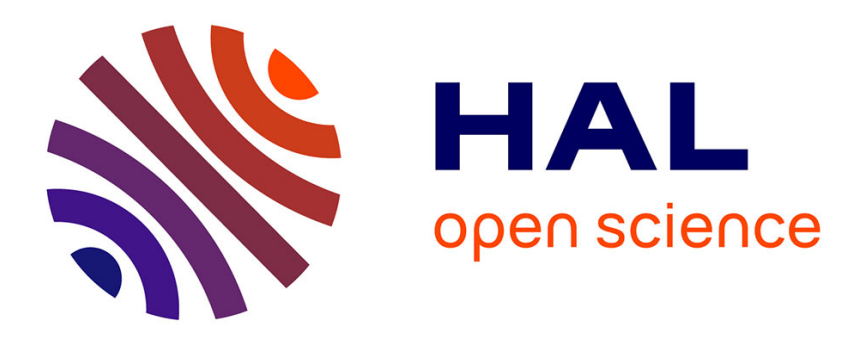

\title{
Diodore de Sicile témoin du Ve siècle av. J.-C. : un âge d'or pour la Sicile?
}

Sophie Bouffier

\section{To cite this version:}

Sophie Bouffier. Diodore de Sicile témoin du Ve siècle av. J.-C. : un âge d'or pour la Sicile?. Dialogues d'histoire ancienne, 2011, supplément 6, p.71-112. hal-00967635

\section{HAL Id: hal-00967635 \\ https://hal.science/hal-00967635}

Submitted on 11 Apr 2014

HAL is a multi-disciplinary open access archive for the deposit and dissemination of scientific research documents, whether they are published or not. The documents may come from teaching and research institutions in France or abroad, or from public or private research centers.
L'archive ouverte pluridisciplinaire HAL, est destinée au dépôt et à la diffusion de documents scientifiques de niveau recherche, publiés ou non, émanant des établissements d'enseignement et de recherche français ou étrangers, des laboratoires publics ou privés. 


\section{Diodore de Sicile témoin du $\mathrm{V}^{\mathrm{e}}$ siècle av. J.-C. : un âge d'or pour la Sicile?}

Dans la préface de sa Bibliothèque Historique, Diodore de Sicile propose de réaliser une histoire universelle, koinè historia, en « un ouvrage qui ramasse, dans les limites d'une seule synthèse, les événements passés dans leur enchaînement » $(\mathrm{I}, 3,8)$.

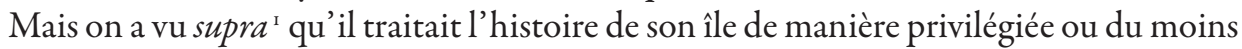
qu' il lui accordait une place importante lorsqu' il traitait certaines périodes. Il était assurément influencé par ses sources, puisque la conception de l'histoire à la fin de l'époque hellénistique ne relevait pas des mêmes exigences que les nôtres. On peut continuer à chercher ce qui relève de Timée, de Philistos, d'Éphore ou des autres en stérilisant un débat arrivé aujourd'hui à son terme vu le peu de parallèles connus. Toutefois, il ne faut pas, à mon avis, oublier que Diodore a opéré un choix et sélectionné ce qui correspondait à son idéologie et ses opinions. Diodore ne s'est pas limité à citer les auteurs qu'il a lus; il s'est documenté sur le terrain et a dû le faire encore plus aisément dans son pays de naissance. Son origine et ses multiples voyages, où il affirme collecter de la documentation ${ }^{2}$, doivent nous inciter à plus de prudence si nous voulons évaluer l'élaboration de sa documentation. Même s'il n'a pas l'ampleur de vue d'un Thucydide ou d'un Polybe, comme on le lui a maintes fois reproché, il ne se contente pas de recopier ce qu'écrivent les autres; il privilégie les versions qui accréditent sa vision de l'Histoire ${ }^{3}$. Dans

\footnotetext{
* Université de Lyon (Lyon 2) - s.bouffier@mmsh.univ-aix.fr Institut de Recherche sur l'Architecture Antique.

I Cf. Introduction, supra, p. I4-16

2 Dans son livre 3, à propos de l'Égypte, il déclare pratiquer l'autopsie hérodotéenne, en se renseignant auprès de ses interlocuteurs locaux, en consultant les archives royales (en particulier 3.38.I). Il suggère avoir fait de même à Rome (I.4.4)

3 Cf. introduction, supra, p. II-12 ; Sacks 1990, ch.4 p. 83-116.
} 
l'évolution de la Sicile, deux époques retiennent l'attention de l'historien et peuvent apparaitre chacune comme un âge d'or : les décennies qui suivent la victoire d'Himère en 480 et le rétablissement de l'ordre et de la paix générale sous Timoléon dans la deuxième moitié du IV siècle.

Je m'intéresserai ici à la première période, celle qui commence avec l'entrée officielle de la Sicile sur la scène internationale et qui se termine avec la prise de pouvoir à Syracuse par Denys l'Ancien, car elle représente une étape décisive dans l'histoire de l'île et de l'Occident grec.

En $48 \mathrm{I}$ av. J.-C., Gélon est sollicité par les Grecs pour une intervention contre les Perses 4 . C'est la première fois dans nos sources que les Égéens font appel aux Siciliens, reconnaissant ainsi la puissance et la prospérité qu'ils ont acquises dès l'époque archaïque, prospérité qu'il nous est difficile d'appréhender autrement que par l'archéologie. Hérodote nous laisse deviner une richesse conséquente mais il la prête au discours d'un tyran qu'il accuse de mégalomanie et qui pourrait ainsi être accusé de grossir exagérément ses forces :

« Je suis prêt à vous secourir avec deux cents trières, vingt mille hoplites, deux mille cavaliers, deux mille archers, deux mille frondeurs et un corps de cavalerie légère de deux mille hommes; et je me charge de ravitailler en blé l'armée grecque tout entière jusqu'à la fin des hostilités s. » (7.158)

L'essor des Grecs de Sicile est à lire également à travers l'allusion du même Gélon à sa victoire dans ce qui paraît être le premier grand conflit gréco-carthaginois :

« Je vous ai demandé, moi, il y a quelque temps de m'aider contre une armée barbare, lorsque je m'opposais aux Carthaginois et que je voulais venger la mort d'Anaxandride, victime des Ségestins, et vous offrais de libérer ces comptoirs qui vous ont procuré tant d'avantages et de profits. [...] le pays, en ce qui vous concerne, pourrait bien être encore aux mains des barbares. N'en parlons plus puisque tout s'est arrangé, à mon avantage ${ }^{6} . \gg(7.158)$

Bien que l'historiographie ne nous en ait rien laissé, les premiers conflits des Sikéliotes contre les Carthaginois se sont soldés par une victoire des Grecs de Sicile, probablement grâce à la puissance militaire de Gélon, rendue possible par l'enrichissement économique et commercial des Sikéliotes.

4 Toutes les dates s'entendent avant J.-C. Les traductions sauf mention explicite sont de l'auteur.

5 Traduction Barguet 1964.

6 Traduction Barguet 1964 .

DHA supplément 6 
Malheureusement le témoignage de Diodore ne nous est pas parvenu pour la période antérieure à 480 , à l'exception de quelques rares fragments ${ }^{7}$. Les premiers temps véritablement représentés chez l'Agyriote (si l'on excepte quelques passages difficiles à remettre en contexte), commencent avec le livre iı. Cette intervention portera donc sur les fragments du livre ı, consacrés à Hippocrate de Géla et à Gélon ${ }^{8}$, et les livres ir à I3 qui embrassent l'ensemble du $\mathrm{V}^{\mathrm{e}}$ siècle av. J.-C.

Le livre ir traitant de la période 480-450 ouvre sur le récit de la Seconde guerre médique auquel fait écho celui de la guerre contre les Carthaginois avec la victoire d'Himère et les conséquences pour les cités grecques de Sicile $(\$ .20-27$; \$. 38). Il retrace également quelques-uns des conflits dans lesquels l'île est impliquée ( $\$ .48$ : affaire d'Himère ; $§ .49$ : bouleversements géopolitiques de Hiéron $\mathrm{I}^{\mathrm{er}}$ en Sicile orientale ; $\$ .5 \mathrm{I}$ : bataille de Cumes ; \$. 66 : l'affaire de Mikythos de Rhégion), la fin de la dynastie des Emménides à Agrigente ( $\$ .53)$, de celle des Deinoménides à Syracuse (\$. 67-68); puis l'installation mouvementée de la démocratie à Syracuse $(\$ .72-73 ; \$ .76)$ cautionnée par l'institution du pétalisme (\$.86-87). Il s'achève sur le mouvement sikèle de Doukétios (\$. 88-92).

Le livre I2, qui traite de la période entre 450 et 415, accorde une place relativement mineure à la Sicile : il met l'accent sur la politique d'expansion territoriale de Syracuse en évoquant la guerre entre Syracuse et Agrigente en 446/445 (\$. 8) et les opérations vers l'intérieur avec la prise et la destruction de Trinaciè en 440/39 (\$. 29-30); les craintes des Léontiniens devant cette politique, à travers l'envoi à Athènes du sophiste Gorgias chargé de plaider pour sa patrie en 427 (\$.53-54). L'intervention athénienne mène à la fin des hostilités, la reconnaissance du droit de cité syracusain aux Léontins et la transformation de leur cité en phrourion syracusain. Dans ce livre se prépare surtout l'interventionnisme athénien dans l'île, avec le récit du conflit entre Ségeste et Sélinonte pour le territoire limitrophe de leur fleuve frontalier et l'appel aux Athéniens par leurs 'frères de race', citoyens de Ségeste et Léontinoi (\$. 82-84).

Le livre 13 retrace les événements entre 415 et 404, du début de l'expédition athénienne de Sicile au début de la Seconde guerre des Carthaginois contre Denys l'Ancien. Les trente quatre premiers chapitres sont entièrement consacrés à l'expédition de

7 Diod., 8, 8-10 : la mort du jeune Actéon dans la tentative de viol par le futur fondateur de Syracuse, le Corinthien Archias. Diod., 8,8-II : l'accusation de détournement de fonds de l'épistate Agathoclès de Syracuse et sa condamnation à mort. Diod., 9, 19 : la fabrication du taureau de bronze par l'artisan Périlaos d'Athènes sur commande du tyran Phalaris d'Agrigente.

8 Diod., IO, 28 : la prise du pouvoir à Géla par Hippocrate en 491. Le pillage des ornements de la statue de Zeus à Syracuse. Diod. 10,29: les cauchemars de Gélon enfant, sauvé par un loup tandis que son école s'effondre lors d'un tremblement de terre. 
Sicile` tandis qu'à partir de 4ro, la relance du conflit entre Ségeste et Sélinonte donne le coup d'envoi de l'expédition d'Hannibal contre les cités grecques à partir de 409 et la prise du pouvoir par Denys l'Ancien ( $\$ .43-44 ; 54-63 ; 75 ; 79-96$; 108-II4).

Entre la bataille d'Himère et l'accession au pouvoir de Denys l'Ancien, la Sicile apparait chez Diodore comme une terre de contrastes : d'abord Eldorado terrestre, comme le soulignent les descriptions de l'économie et de la société sikéliotes, mais également lieu de conflits politiques et ethniques où l'historien met en place l'image d'une grécité soucieuse de s'affirmer contre toute tentative de « contamination » barbare ${ }^{10}$. J'essaierai d'étudier dans cette intervention cette bivalence de l'image donnée par l'historien sur sa terre natale, et me concentrerai sur trois champs thématiques principaux : politique, économique, culturel.

\section{Vers l'unité politique?}

C'est probablement le champ thématique où le témoignage de Diodore a été le plus exploité et ce pour des raisons historiographiques (l'histoire politique ayant longtemps compté plus que les autres). Diodore est l'un des rares historiens à donner des informations sur l'évolution politique de la Sicile. Ainsi sur les différents régimes politiques, en particulier les tyrannies des Deinoménides et des Emménides. L'analyse de N. Luraghi en 1994 est la plus complète et pertinente faite à ce jour et il n'y a pas lieu d'y revenir. Je me limiterai à mettre en exergue quelques traits marquants du témoignage diodoréen.

I. la tyrannie : alors que la Grèce égéenne a renversé les tyrannies, cette forme de régime prend en Sicile une ampleur qui ne s'achève qu'avec la victoire de Rome en 2I2/II. Diodore évoque surtout celle des Deinoménides et des Emménides, négligeant en grande partie celles des autres cités, notamment Rhégion ou Himère. La vision que reflète Diodore est-elle celle que l'on observe dans ses sources ${ }^{11}$, souvent hostiles à la tyrannie, qu'elles soient issues du courant athénien ou des factions locales défendant les aristocraties sikéliotes ? ou peut-on la qualifier d'originale ? L'historien utilise des termes assez neutres pour désigner ces régimes. Théron est nommé plus souvent

9 Les paragraphes 34 et 35 offrent une digression sur l'action politique du législateur Dioclès à la fin de l'expédition athénienne.

Io Voir dans ce même volume, Péré-Noguès, infra, p. 9 ; Cusumano, infra, p. 9 ; Cohen-Skalli, infra, p. 9.

II On y a vu là l'influence d'Éphore et de Timée (Meister 1967, p. 43). 
'dunastès' (II, 20, 5; II, 53, I) que 'turannos' (II, 48, 5) des Agrigentins ${ }^{12}$. Le gouvernement de Gélon et ses successeurs est une basileia ${ }^{13}$. Le terme de 'turannos' est appliqué à Anaxilas de Rhégion (II, 48, 2) puis à Denys l'Ancien à la fin du V $V^{e}$ siècle. La dynastie des Deinoménides apparaît comme le paradigme de l'idéologie diodoréenne. L'historien dresse un panégyrique de Gélon, à travers la stratégie adoptée à Himère puis sa politique générale jusqu'à sa mort en 478 : euergétés, soter, basileus sont les titres que lui attribue Diodore et qui ne sont guère employés au début du $\mathrm{V}^{\mathrm{e}}$ siècle en Grèce égéenne, sinon pour des dieux ou des souverains barbares. On pourrait s'interroger sur l'éventuelle contamination qu'ont pu opérer sur le langage de l'historien les régimes hellénistiques où ces titres couronnent traditionnellement les souverains. Mais si pour les deux premiers termes, euergétés et soter ${ }^{14}$, on peut mettre en doute la véracité du récit diodoréen, pour l'appellation de basileus, il nous informe très vraisemblablement de la forme constitutionnelle prise par le régime des Deinoménides. Les honneurs du fondateur qui leur sont rendus à leurs funérailles font partie, selon moi, du protocole de la basileia mise en place par Gélon. De même l'historien insiste sur les grandes qualités militaires ${ }^{15}$ de Gélon, notamment son audace et son sens de la stratégie, sa piété, qu'il associe à la générosité, la magnanimité, toutes qualités qui composent la clementia romaine et qui, dans la pensée diodoréenne, en font un précurseur des grands personnages de l'histoire, avant Alexandre, Timoléon ou Jules César. Le personnage de Gélon entre dans la catégorie de ces héros qui selon Diodore contribuent au progrès de l'humanité, comme l'avait mis en lumière K.S. Sacks ${ }^{16}$. Mais la tyrannie elle-même n'est pas critiquée sauf dans le cas des abus des descendants de Théron et Gélon. Diodore reprend ainsi un topos de l'historiographie grecque d'époque classique. Hiéron, frère et successeur de Gélon, « aimait l'argent, était violent et d'une façon générale était tout à fait étranger à la simplicité de mœurs et la noblesse d'âme de son frère. » (II.67). Thrasybule, le dernier des Deinoménides, « qui avait reçu une royauté établie sur des solides fondations, perdit honteusement le pouvoir à cause de ses vices. » (II.68). Dans tous les cas, qu'il s'agisse des Deinoménides, des Emménides, de Denys et de son successeur, les descendants n'ont pas mérité leur pouvoir et sont donc responsables de leur chute. Pour

\footnotetext{
I2 Van Compernolle R. 1992.

I3 II, 23, 3; II, 38, 3; II, 66, 4. Le terme de basileus est employé en II, 48,3; II, 5I,I ; II, 66, I ; II, 66, 4 ; II, 67, I. Les traductions adoptées ici pour le livre in sont celles de J. Haillet, CUF, sauf mention explicite.

I4 Selon K.S. Sacks, l'association des deux termes est récurrente dans l'évocation des bienfaiteurs de l'humanité tels que les conçoit Diodore, qu'il s'agisse de personnages mythiques ou légendaires (Sacks 1994, p. 214). Il s'agit d'une figure de style plutôt que d'un emprunt à Timée comme on a voulu l'interpréter. I5 II, 22-26.

I6 Sacks 1990, p.6I-78; puis Ambaglio 1995, p. I09-II8.
} 
Diodore, le pouvoir monarchique n'est pas un mauvais régime en soi, contrairement à ce que l'on voit chez Hérodote, lorsqu'il présente l'ambassade des Grecs à Syracuse avant l'attaque perse de 480 ; et la démocratie n'est pas considérée comme le meilleur des régimes. Le régime en lui-même n'est pas condamnable : c'est ce que les gens en font qui peut être critiquable. Telle est l'interprétation que l'on peut tirer de sa présentation de la démocratie syracusaine au lendemain de sa promulgation : il laisse entendre que Syracuse se veut une nouvelle Athènes, comme le montrent les mesures prises par le nouveau régime. La chute de la tyrannie entrâne l'institution d'une cérémonie de commémoration, les Eleutheria, constituée d'un sacrifice annuel de 450 taureaux, d'un banquet public et de jeux solennels (II.72), puis la loi du pétalisme qui imite l'ostracisme athénien, mais qui est rapidement abrogée sous prétexte que les kaloi kagathoi se détournent de la vie publique à cause des démagogues et des sykophantes :

« Les hommes les plus puissants étant frappés de cet exil, les citoyens les plus distingués, qui étaient les plus capables par leur mérite personnel d'apporter des améliorations aux affaires de l'État, se détournèrent de la vie publique et, à cause de la crainte que leur inspirait cette institution, ils se contentaient de vivre en simples particuliers et, occupés de leur seule fortune personnelle, ils tombaient dans la mollesse, tandis que les citoyens les plus malfaisants et les plus impudents s'occupaient des affaires publiques et poussaient les masses au trouble et à la révolution. De nouvelles luttes civiles se produisirent donc, la masse du peuple s'abandonnait à des dissensions, de nouveau la ville tomba dans des troubles incessants et graves. La foule des démagogues et des sycophantes exerçait une influence prépondérante, la jeunesse ne cultivait que l'habileté dans l'art de la parole et, d'une manière générale, beaucoup avaient remplacé par une conduite perverse le genre de vie honnête d'autrefois et si, grâce à la paix extérieure, les fortunes étaient prospères, on ne se souciait guère de concorde et de respect de la justice. » (II.87)

Ce que nous dit Diodore de la démocratie syracusaine montre bien que le type de régime n'a pour lui aucune importance; il suffit que les notables s'investissent dans la vie civique. En réalité, les doctrines politiques n'intéressent pas Diodore. On le remarque par exemple dans la faible part qu'il accorde à la présentation du régime agrigentin après la chute de Thrasydée en 466. Les données à notre disposition proviennent d'autres sources, notamment Diogène Laerte ${ }^{17}$. De même les informations que l'Agyriote emprunte à Timée concernent le mode de vie des Agrigentins, pas leur régime politique, et comme on l'a bien montré, il s'agit plutôt d'un gouvernement oligarchique modéré que d'une démocratie comparable à celle d'Athènes ou de Syracuse. C'est l'éthique qui importe à Diodore et qu'il met en exergue dans l'introduction de la

17 Asheri 1992.

DHA supplément 6 
Bibliothèque Historique ${ }^{18}$. L'histoire est pédagogique à travers le portrait de ses grands hommes. Ainsi l'image du simple particulier qu'il véhicule dans le portrait des grands chefs est plus tributaire de l'idéologie républicaine romaine que de l'idéologie démocratique grecque. Le milieu politique et intellectuel où évolue Diodore est loin d'être étranger à ses choix idéologiques. Diodore vit la fin de la république romaine, le déclin des institutions traditionnelles et la montée d'hommes forts, au premier rang desquels figure Jules César pour lequel il ne cache pas son admiration ${ }^{19}$. On a souvent mis en lumière le césarisme de Diodore, visible dès le traitement particulier attribué à Héraclès, ancêtre de la gens Julia ${ }^{20}$ ou dans le récit de la bataille d'Himère ${ }^{21}$, qui montre Gélon comme un précurseur de César. Son désintérêt total pour l'intervention politique d'un personnage comme Empédocle, par exemple, qu'il ne cite même pas dans son tableau $\mathrm{du} \mathrm{V}^{\mathrm{e}}$ siècle sicilien, est selon moi emblématique de la vision apolitique de l'historien.

2. Une époque de remaniements géopolitiques et géostratégiques importants. Avec la bataille d'Himère en 480 , s'ouvre une ère de bouleversements qui s'achève avec la conquête romaine au III siècle. Pour Diodore, Himère est un combat des « Grecs de Sicile » contre les Carthaginois (II.20.I), pour la défense des Grecs contre les barbares, rassemblés en une coalition punico-perse ${ }^{22}$. On a voulu reconnaître dans sa présentation l'influence de Timée et attribuer à l'historien de Tauroménion le chauvinisme dont il fait preuve ${ }^{23}$ lorsqu'il relaie la thèse que Gélon remporta la victoire à Himère le jour où Léonidas et ses Spartiates tombèrent aux Thermopyles ${ }^{24}$ et que la victoire de Gélon donna aux Grecs le courage d'affronter les troupes perses d'autant que pas un seul des chefs carthaginois ne rentra à Carthage :

« Il faut dire que les habitants de la Sicile comme ceux de la Grèce éprouvèrent, avant le combat, la plus grande frayeur devant la masse des forces barbares mais la victoire de Gélon et des Sikéliotes, intervenue la première, donna confiance aux Grecs de Grèce quand il l'apprirent ; de plus, pour

I8 Voir en particulier, Chamoux et Bertrac 1993, XV-XVIII ; LVI-LX. Sachs 1990, Ambaglio 1995, pour qui le moralisme de Diodore est surtout perceptible dans les livres 2I à 40 de la Bibliothéque Historique.

19 Ambaglio 1995, p. 13-16.

20 Ambaglio 1995.

2 I Devillers 1998.

22 II.2O.I : « Les Carthaginois étaient convenus avec les Perses de soumettre au même moment les Grecs de Sicile ».

23 Meister 1967, p. 42-43; Pearson 1987, p. 138-140.

24 II.24.I : « Il se trouve que le jour où Gélon remporta la victoire coïncida avec la lutte qu' aux Thermopyles Léonidas et ses hommes soutinrent contre Xerxès, comme si la divinité avait voulu expressément qu'eussent lieu en même temps la plus belle victoire et la plus glorieuse défaite. » 
ce qui est des hommes qui, dans les deux armées, exercèrent le commandement suprême, du côté perse, tandis que le Roi réussit à s'enfuir et des centaines de milliers d'hommes avec lui, du côté carthaginois, non seulement le général fut tué, mais encore tous ceux qui participaient avec lui à cette guerre furent massacrés, au point qu'il n'y eut pas même un messager à ce qu'on dit, qui réussît à gagner Carthage sain et sauf » (II.23.2)

Peut-être également tributaire d'Éphore, défenseur d'un panhellénisme à l'échelle méditerranéenne, notre historien oriente les projecteurs sur les personnages d'Amilcar et Gélon, rejetant dans l'ombre « les autres », ses ennemis siciliens qu'il évacue d'une demi-phrase « « les cités et les souverains qui avaient été autrefois ses adversaires », et ses alliés qu'il démobilise à la fin de la guerre (II.25); parmi les alliés on sait qu'il se trouvait au moins Théron d'Agrigente dont l'attitude telle qu'elle est décrite par Diodore pendant cette guerre n'a rien d'hérö̈que. On l'a déjà souligné, le portrait de Théron est un portrait en creux de celui de Gélon, probablement orchestré et mis en scène par une propagande habile. Or la politique menée par les Agrigentins depuis Phalaris, et les Géloens depuis la tyrannie d'Hippocrate, est dirigée essentiellement contre les Eubéens qui tiennent le détroit de Messine et les routes commerciales entre le monde grec oriental et la mer Tyrrhénienne. Théron, depuis Agrigente, et Gélon, depuis Syracuse, ont poursuivi l'expansion territoriale de leurs prédécesseurs. La bataille d'Himère est un événement supplémentaire dans l'affrontement des réseaux de l'époque archaïque qui modifient définitivement la carte des dominations territoriales sikéliotes. Dans un second temps, la politique menée par la coalition aboutit à l'élimination du réseau eubéen, désorganisé par les synœecismes de Gélon (destruction de Léontinoi, rassemblement de la population de la Sicile orientale à Syracuse) et les fondations urbaines de son successeur Hiéron : fondation de la ville d'Aitna, expulsion des Catanéens et des Naxiens, repeuplement de la région avec des Péloponnésiens et des Syracusains et partage de leur chôra entre ces nouveaux colons. La Catane eubéenne est devenue une Aitna péloponnésienne que Thucydide qualifierait de dorienne, comme Géla, Agrigente et Syracuse. Selon l'interprétation un peu étroite de Diodore, Hiéron veut des auxiliaires en cas de danger pour son pouvoir et vise l'institution des honneurs héroïques dus aux fondateurs d'une ville de dix mille habitants, alors qu' il s'agit, comme je le précisais supra d'une vraisemblable prérogative du régime institué par son frère.

En réalité, sans l'affirmer consciemment, l'Agyriote met l'accent sur la réorganisation géostratégique de l'île qui mène à une forme d'unité insulaire. Les Grecs de Sicile deviennent les Sikéliotes au détriment des Sikèles, Carthaginois et autres barbares. L'affaire de Doukétios au milieu du $\mathrm{V}^{\mathrm{e}}$ siècle apparaît, dans ce contexte, à la fois comme la preuve d'une politique d'union sikèle anti-grecque, et comme le dernier avatar du conflit Doriens-Eubéens, puisque jusqu'au premier quart du $\mathrm{V}^{\mathrm{e}}$ siècle, les Eubéens 
maîtrisaient les relations avec les Sikèles. Toute la politique extérieure de Syracuse en Sicile, à partir de Gélon, est destinée à créer puis à consolider cette unification sous sa coupe, quel que soit le type de régime auquel elle est soumise. Cette orientation de l'action syracusaine semble poursuivie par les successeurs des Deinoméinides comme il transparaît de la législation de Dioclès dont Diodore nous dit qu' « elle était en vigueur dans de nombreuses cités de Sicile jusqu'au moment où tous les habitants de la Sicile devinrent citoyens romains » (13.35). La domination syracusaine s'est faite militairement avant de prendre la forme d'une politique de propagande idéologique. Ainsi pour le $\mathrm{V}^{\mathrm{e}}$ siècle, l'historien évoque l'installation de phrouria dans le quart sud-est de la Sicile lors du mouvement de Doukétios ${ }^{25}$. Il ne les nomme pas, à l'exception du site de Motyon ${ }^{26}$ pris par les Agrigentins. Les recherches archéologiques menées dans cette région ont mis en évidence l'existence de sites, fortifiés dans le courant du $\mathrm{V}^{\mathrm{e}}$ siècle, comme celui de $\mathrm{Mineo}^{27}$ ou les établissements du système fluvial Gornalunga- Fiume del Margi ${ }^{28}$, tandis qu' un certain nombre d'autres sites, subissaient des destructions, tels Monte San Mauro, Morgantina ${ }^{29}$ ou Montagna di Ramacca ${ }^{30}$, Monte Balchino dans le territoire de Caltagirone ${ }^{31}$, peut-être Centuripe ${ }^{32}$. L'historiographie y a souvent vu la preuve sur le terrain de la rivalité entre les Eubéens et l'alliance syracuso-agrigentine, en attribuant la plupart de ces sites à la sphère d'influence chalcidienne ${ }^{33}$. Il faut envisager l'existence de deux types d'établissements, ceux qui ont pu être créés ex-nihilo ou ceux qui ont dû renforcer les défenses d'établissements indigènes alliés. La dernière

25 Ir.9I.3-4 : les phrouria des Sikèles dont celui de Motyon. En 45I-50, après la défaite des Sikèles face aux Syracusains, « La plupart de ceux qui échappèrent au massacre se réfugièrent dans les forts des Sikèles et sauvèrent ainsi leur vie. [91.5] Pendant ces événements, les Agrigentins prirent d'assaut le fort de Motyon, que tenaient les Sikèles restés avec Doukétios... »

26 La plupart des historiens l'identifient à Vassallaggi-S.Cataldo : voir Asheri 1992, note I4. Selon Miccichè 1989, il s'agit de Sabucina.

27 Belfiore 2000. Le site de Mineo (Piano dei Casazzi) fut d'abord un site indigène, fortifié probablement dès le $\mathrm{VI}^{\mathrm{c}}$ siècle puis renforcé au début du $\mathrm{V}^{\mathrm{e}}$ siècle.

28 Sjöqvist 1962 ; Vallet 1962

29 Sjöqvist 1962.

30 Procelli et Albanese 1992.

3I En dernier lieu avec bibliographie précédente, Lamagna 1993-94.

32 Biondi 2002 : l'occupation du site n'est plus attestée entre l'époque archaïque et l'époque timoléonienne.

33 L'exemple de Monte San Mauro est un des exemples de la difficulté, voire de l'inutilité de ce débat : traditionnellement rattaché à la sphère d'influence géloenne, il a été également attribué à l'aire chaldidienne : en dernier lieu, voir Greco 1999a. M. Frasca (1997) l'identifie au site d'Euboia, avant-poste de Léontinoi. Sur les problèmes d'hellénisation de la Sicile sud-orientale, cf. Procelli 1989. 
péripétie de cette unité sikéliote est le mouvement de Doukétios ${ }^{34}$ qui scelle la disparition de l'antagonisme Doriens-Eubéens en le renvoyant au passé mais voit émerger un nouveau rapport de forces, la rivalité Syracuse-Agrigente ; larvée pendant les régimes de Théron et de Gélon, celle-ci apparaît en filigrane dans l'œuvre diodoréenne et prend fin seulement avec l'expédition carthaginoise de 409/405.

3. L'élaboration d'une unité culturelle sikéliote des Grecs de Sicile nous amène au dernier point qu'il me paraît essentiel de souligner dans la vision diodoréenne du $V^{e}$ siècle sicilien : l'importance des étrangers : les $x e n o i^{35}$, que j'entends ici dans son sens le plus large et non pas au sens restreint des mercenaires. Les étrangers, ce sont à la fois des indigènes (les villes sikelikai) et des mercenaires ou des colons grecs venus de l'extérieur de l'île, comme les Péloponnésiens du peuplement d'Aitna. La question apparaît à ce moment-là et va marquer tous les rapports sociaux et la vie politique de la Sicile jusqu'à Hiéron II au III $^{\mathrm{c}}$ siècle. C'est une question fondamentale car elle est liée à celle du partage des terres. Les tyrans ont introduit, dans le corps civique de leur cité ou de celles qu' ils avaient sous leur domination, de nombreux mercenaires ou nouveaux colons qui, à l'instauration de la démocratie, ont été déchus de leurs droits politiques quand ils n'ont pas été chassés des terres qu'on leur avait données ${ }^{36}$. Diodore l'évoque en particulier à propos des soldats de Gélon (II. 72.3). Ces mercenaires semblent apparaître dans certaines cités, notamment à Géla, où, comme l'a suggéré l'intéressante lecture de J. de La Genière ${ }^{37}$, une fois obtenus leur reconnaissance civique et des biens matériels, ils se faisaient enterrer dans les mêmes nécropoles que les Géloens d'origine, avec un rituel et un apparat quelquefois différents.

\section{Un âge d'or économique et social}

Diodore insiste sur l'essor économique de la Sicile qui s' instaure après la bataille d'Himère. Il l'attribue à l'afflux de butin et de main d'œuvre bon marché. Ainsi :

\footnotetext{
34 Sur le sujet, voir Péré-Noguès infra.

35 Sur les mercenaires, Tagliamonte 1994.

36 Voir infra, p. 85-87; également à ce sujet Asheri 1992, en particul. p. 99-100; Collin Bouffier 2010.

37 La Genière 2003 : certains vases de la nécropole de Géla, datés du second quart du $\mathrm{V}^{\mathrm{c}}$ siècle, suggèrent en effet leur présence. On y retrouve des formes et une iconographie régulièrement choisies par les potiers athéniens à destination d'une clientèle étrusque et campanienne, comme les amphores de Noles ou les stamnoi à figures rouges. De même La Genière 1999.
} 
« Les Agrigentins avaient aussi profité de la fertilité du terrain pour y planter des vignes et toute espèce d'arbres dont ils tiraient de grands revenus ${ }^{38}$. » (II.25)

La Sicile jouit également de la période de paix qui s'instaure après la chute des tyrannies :

« En Sicile, une fois que la tyrannie fut renversée à Syracuse et que toutes les cités de l'île furent libérées, la Sicile toute entière fit aussitôt des progrès considérables vers la prospérité. En effet, grâce à la paix, les Sikéliotes qui habitaient un pays fertile, retrouvèrent rapidement leurs richesses grâce à l'abondance de la production agricole, remplirent le pays d'esclaves, de troupeaux et de toute sorte de biens et accrurent leurs revenus, les richesses n'étant plus absorbées par les guerres répétées ${ }^{39}$. $\gg(11,72)$

Diodore récapitule enfin la prospérité des Agrigentins à la fin du $\mathrm{V}^{\mathrm{e}}$ siècle, sorte de paradigme de l'âge d'or sicilien :

« Les Agrigentins, dont le territoire jouxtait l'épicratie carthaginoise, comprirent qu'ils sentiraient les premiers le poids de la guerre, et c'était le cas. En conséquence, ils résolurent de transporter depuis la campagne à l'intérieur des murailles le grain, les fruits et tous les autres biens. À cette époque, le territoire et la ville des Agrigentins regorgeaient de richesses : il ne me semble pas hors de propos d'en communiquer quelques détails. Aucun pays ne produisait des vignobles plus étendus et plus beaux. La plus grande partie du territoire était plantée d'oliviers, dont le fruit s'exportait et se vendait à Carthage, car en ce temps-là, la Libye n'était pas encore cultivée. Les Agrigentins, recevant de l'argent en échange de leurs produits naturels, amassèrent une richesse incroyable. $\gg(13.8 \mathrm{I})$

L'intérêt de Diodore pour l'économie s'arrête à des considérations très générales dont on peut tirer néanmoins quelques remarques avant de s'interroger sur la validité du témoignage au vu des découvertes archéologiques. D'abord, et on l'avait déjà remarqué ${ }^{\circ}$, pour Diodore, la prospérité économique est indissociable de l'essor démographique : à la richesse de la production correspond une population nombreuse et dense ; Agrigente combine ainsi 'richesse' et 'polyanthropia'. Ensuite de manière implicite, l'Agyriote lie la propriété foncière à la rentabilité de la production. Il nous offre une vision statique et sans relief de la l'évolution économique. Alors que le siècle aurait vu maintes révolutions agraires, la prospérité se serait maintenue sans variations. Dans le détail, que peut-on tirer des informations diodoréennes?

Sur les modes d'exploitation de ces campagnes, Diodore reste muet, comme tant d'autres avant lui. Quelle forme de paysage s'offrait au visiteur ? Quel type de culture

38 Traduction personnelle.

39 Trad. Haillet remaniée.

40 Gallo 1989. 
caractérisait telle ou telle cité ? Parmi les sources littéraires, seul Thucydide nous livre quelques informations lors du récit de l'expédition de Sicile et les rares témoignages épigraphiques disponibles décrivent un paysage d'époque hellénistique ${ }^{41}$. Alors qu'elles opèrent leur retraite vers le sud de la côte syracusaine en 4I33, les troupes athéniennes traversent les champs syracusains entourés de murets ${ }^{42}$, comme on peut le voir encore aujourd'hui où les plantations d'agrumes ont remplacé en maints endroits les oliviers et autres cultures arbustives antiques.

Si l'on cherche du côté de l'archéologie, il faut bien reconnaître que les recherches spécifiques à la Sicile sont encore limitées alors que l'Occident a été l'un des champs pionniers de l'étude des territoires antiques. Jusqu’à présent, faute de véritable investigation, les territoires des grandes cités évoquées par Diodore, Syracuse ou Agrigente ont révélé peu de traces de cette exploitation intensive. Quelques régions ont fait l'objet de prospections plus poussées, comme celle de Camarina ${ }^{43}$ ou celle, exemplaire, d'Himère ${ }^{44}$.

Étant donné que la Sicile était consacrée à Déméter ${ }^{45}$ comme l'atteste Diodore dans le livre 5 de la Bibliothéque Historique, et qu'elle exportait du grain vers le Péloponnèse et vers $R_{0}{ }^{46}$, les céréales devraient représenter une large part de la production agricole. Or pour le $V^{c}$ siècle, si l'on excepte l'exemple surexploité de Camarina ${ }^{47}$, elles sont relativement peu attestées. Dans une des tours de la fortification d'époque classique, A. Di Vita avait découvert une réserve de grains stockés et brûlés en 405 lors du siège des Carthaginois. Les tables d'Entella, qu'on a l'habitude d'utiliser, attestent une situation d'époque hellénistique ${ }^{48}$.

Dans l'arrière-pays de Sélinonte, la riche vallée du Belice, lieu de pénétration des colons mégariens vers l'arrière-pays, a révélé la présence de meules céréalières qui prouvent non seulement l'activité céréalière de la région, mais aussi le probable

4I Les tables d'Halaisa sont une source précieuse, bien que leur publication définitive soit encore en chantier : cf. Prestianni Giallombardo 1998.

42 Thuc, 7.81.4

43 En dernier lieu, Di Stefano G. 200 I.

44 En dernier lieu, Belvedere 200I. À Sélinonte ou Syracuse, les résultats de F. Lentini ou de L. Guzzardi sont encore indisponibles. Pour les sites de Morgantina, de Troina, ou d'Entella, on dispose seulement de quelques premières notices: Morgantina, Thompson 2001; Thompson 2002 ; Troina : Malone, Ayala et al. 200I-2003; Entella, cf. la bibliographie de De Angelis 2006a, p. 177. Le territoire de Kentoripai a également fait l'objet de prospections encore peu exploitées : Biondi 2002.

45 Di Stefano C.A. 2008 ; Hinz 1998.

46 Cf. Gallo 1992; Gallo 1989; Fantasia 1993; Nenci 1993 ; De Angelis 2001.

47 Di Vita et Costantini 1983.

48 Entella 2001.

DHA supplément 6 
commerce de ce produit. Sur le site de Monte Castellazzo di Poggioreale ${ }^{49}$, dans des couches datées du VI ${ }^{\mathrm{e}}$ au début du $\mathrm{IV}^{\mathrm{e}}$ siècle, on a mis au jour des meules non seulement dans des habitations, mais également dans un espace mal identifié, où leur concentration incite à supposer l'existence d'un véritable établissement, voire d'un quartier spécialisé dans la meunerie. Outre donc une activité domestique réservée à l'approvisionnement domestique, on peut envisager une structure de production destinée à la vente. Le type de meule découvert, la meule rotative, encore peu répandu dans le monde grec ${ }^{50}$, renforce l'hypothèse d'une activité de production organisée. Enfin le matériau d'une partie de ces meules suppose une intégration du site dans les circuits commerciaux insulaires, voire internationaux. Outre les roches sédimentaires locales, certaines d'entre elles sont taillées dans un basalte provenant de Sicile orientale, ainsi que de la zone tyrrhénienne (Campanie, Sardaigne et Étrurie) et même probablement de l'aire égéenne. Que l'on ait importé les objets eux-mêmes ou leur matériau ${ }^{\text {sI }}$, ces découvertes montrent une capacité d'organisation et de transformation de la production agricole qui conforte les informations diodoréennes sur la situation agraire de l'île.

On ne connaît néanmoins pour cette époque aucune structure de stockage destinée à un commerce à grande échelle. Les Sikéliotes utilisaient couramment des structures multifonctionnelles comme les portiques, ou les fortifications, voire les demeures particulières, réquisitionnées ad hoc en cas de guerre. J'ai proposé, dans un article sous presse, de chercher des structures aériennes en bois, matériau abondant en Sicile et facilement accessible depuis les grandes zones de production céréalière ${ }^{52}$. C'est la typologie que préconise Philon d'Alexandrie au III ${ }^{e}$ siècle av. J.-C.

On trouve en Sicile d'autres types de productions qui témoignent d'une production destinée d'abord à l'approvisionnement local avant d'être exportée. Ainsi, le territoire d'Himère, qui a fait l'objet de prospections systématiques, nous offre un tableau précis des conditions d'exploitation du territoire : l'existence d'unités agraires fondées sur des systèmes mixtes agriculture (céréaliculture et arboriculture) /élevage, et sur l'exploitation de la forêt dans un milieu géomorphologique et paysager diversifiés Ce mode d'exploitation a été confirmé par la découverte, dans la ville elle-même, d'un important dépôt d'outils agricoles, qui associe des outils utilisés principalement pour la culture des céréales, comme le soc et la pioche ou pour le binage des cultures arboricoles, comme la houe à deux dents, ou pour la coupe du bois comme la hache. Les analyses

\footnotetext{
49 Spatafora 1993.

50 Amouretti 1986, p. 144-I47.

5I Les découvertes sous-marines ont confirmé le commerce de meules : cf. Beltrame et Boetto 1997.

52 Laudani, Collin Bouffier et al., sous presse.

53 Alliata et Belvedere I988; et l'excellente synthèse de Belvedere 200I.
} 
géomorphologiques et géoarchéologiques, qui ont mis en évidence un progressif réchauffement climatique de la zone, ont permis d'observer que la cité était bordée, sur la bande côtière, de zones marécageuses, soumises à la mobilité des fleuves Torto et Himère. Ainsi, la plaine de Buonfornello constituait une zone propice aux activités spécifiques aux zones humides : pêche, chasse, exploitation des plantes de marais. Les Himéréens ont privilégié un accès direct aux ressources hydriques et au maquis, les ressources forestières offrant le double avantage de fournir du pâturage régulier aux troupeaux et du bois comme matériau de construction ou de chauffage. La présence de plaines côtières à tendance lagunaire n'est pas l'apanage d'Himère. Syracuse, Géla ou Camarina présentent les mêmes caractéristiques géomorphologiques, offrant ainsi des conditions très favorables pour l'élevage, notamment des chevaux dont on connaît l'importance dans la société sikéliote ${ }^{\varsigma 4}$. Dans l'arrière-pays, la forêt, qui recouvrait la zone collinaire jusqu'à la ligne de partage des eaux et sur les pentes du Monte Roccelito, se prêtait bien à une exploitation pastorale saisonnière (estivale), l'élevage étant indispensable à un système agricole à haute intensité de travail, tel que les prospections de l'équipe palermitaine l'ont suggéré.

Dans le territoire d'Agrigente, Diodore évoque non pas la fonction céréalière d'Agrigente, mais la rentabilité de sa production arbustive (vigne et huile d'olive), qui nécessite une présence régulière pour les diverses façonsss à appliquer à ces arbres, et qui devrait donc laisser des traces sur le terrain, décelables uniquement par des fouilles attentives. La présence de la viticulture est peut-être suggérée d'ailleurs par la popularité des thèmes dionysiaques dans la céramique attique importée à Agrigente depuis la fin du $\mathrm{VI}^{\mathrm{e}}$ siècle ${ }^{56}$. Pour l'instant, les rares prospections menées sur la côte entre Agrigente et Siculiana ${ }^{57}$, ont révélé une majorité de sites antérieurs à la fin du $\mathrm{V}^{\mathrm{e}}$ siècle, tous localisés à moins de dix kilomètres à vol d'oiseau de la ville rhodio-crétoise. Les archéologues ont supposé que cette zone, qui offre des conditions relativement médiocres d'exploitation agricole, a pu être consacrée à l'élevage, sans toutefois avancer de testimonia précis. Le seul site qui semble avoir quelque importance et perdurer durant toute la période jusqu'à l'époque romaine est celui de Faro Rossello, situé à onze kilomètres à l'ouest d'Agrigente, sur la côte au débouché de la petite vallée fluviale du torrent Forte qui représente une voie d'accès vers l'arrière-pays. Qu'on l'interprète comme un phrourion,

54 Outre le rôle de la cavalerie dans les guerres, en particulier celle de Gélon à Himère (II.), l'iconographie céramique des vases découverts à Géla ou à Agrigente conforte l'importance de cette activité dans le mode de vie des élites : cf. Collin Bouffier 1998 ; à Géla, Panvini et Giudice 2003 ; à Agrigente, Deorsoli et al. 1988, T.1087, p. 320 ; T.936, p. 326 ; T.111, p. 340-341 ; 697 p. 342-343, 22, p. 344-345 ; T.971, p. 349.

55 Amouretti 1986, p. 5I-75; 200-204; Nenci 1993.

56 Veder greco 1988.

57 Di Bella et Santagati, 1998.

DHA supplément 6 
du fait de sa situation stratégique, ou comme un emporion consacré à l'embarquement des marchandises du territoire, du fait de sa baie abritée, le site de Faro Rossello ${ }^{58}$ est un jalon de l'organisation et de l'exploitation territoriales agrigentines.

Ailleurs, les traces de plantations découvertes récemment au Plemmyrion à Syracuse, ou dans le territoire de Camarina, pourraient bien remonter à l'époque classique, même si les fosses mégariennes mises au jour au sud de la fortification à Mégara Hyblaea semblent plutôt d'époque hellénistique. On a pu mettre en évidence également la pratique régulière de l'irrigation dès l'époque classique ${ }^{59}$, comme à Camarina ${ }^{60}$, qui reflète une politique d'amélioration de la production.

La production agraire de la Sicile au $V^{\mathrm{e}}$ siècle apparaît donc comme plus diversifiée qu'on n'a voulu le dire ${ }^{61}$ et il faut probablement distinguer des types et des niveaux de production selon les régions. Même dans les territoires ${ }^{62}$ qu'on a pu évaluer au-dessus des $500 \mathrm{~km}^{2}$, on ne peut parler de culture spécialisée, voire 'spéculative', si l'on nous concède ce terme anachronique. Dans une économie marquée par la difficulté des périodes de soudure ${ }^{63}$, le premier objectif est de fournir le nécessaire, le surplus étant ensuite commercialisé. Les inscriptions d'Entella ${ }^{64}$ montrent bien qu'il en était ainsi au moins à l'époque hellénistique et que la Sicile n'était pas à l'écart des grandes crises alimentaires qui frappaient régulièrement le monde grec. Comme en Grèce égéenne, les Sikéliotes ont ainsi dû multiplier les espèces cultivées ; la différence entre les deux régions réside non pas tant dans la qualité que dans la quantité, l'étendue des terres disponibles multipliant les capacités de rendements.

En ce qui concerne l'organisation de la propriété foncière, le $\mathrm{V}^{\mathrm{c}}$ siècle sicilien apparait, chez Diodore, comme une période révolutionnaire : ainsi l'attestent les bouleversements démographiques et géopolitiques mis en œuvre d'abord par les tyrans, puis par les gouvernements qui leur ont succédé. Les premiers auraient confisqué des terres à leurs opposants pour les donner à leurs mercenaires ou à de nouveaux immigrants (ainsi à Catane, Naxos, Himère, Géla, ou Léontinoi), politique qu'auraient abolie les seconds en procédant à un rappel général des exilés, à un nouveau partage des terres

58 Certains historiens locaux ont voulu l'identifier à Herbessos citée par Polybe (I, 29): cf. Di Bella et Santagati, 1998 , p. 80.

59 Collin Bouffier 2008.

60 En dernier lieu Di Stefano G. 200 I et bibliographie antérieure.

6I Sur la surévaluation du grain dans l'économie sicilienne, cf. De Angelis 2006b.

62 Hansen 2006a, appendix 4.

63 Garnsey 1996, p. 27-74.

64 Entella 200I. 
et à une redistribution des charges et fonctions politiques. Cette politique ne fait que commencer en Sicile et se poursuit sous Denys l'Ancien et Timoléon et pendant toute l'époque hellénistique ${ }^{65}$. En l'espace de trente ans, les terres auraient changé de mains au moins deux fois. Les mutations ne se font pas sans conflits, comme le souligne Diodore en décrivant les diverses étapes du processus. Après un premier partage entre 480 et 467, lors des gouvernements de Théron à Agrigente, Gélon et Hiéron Ir à Syracuse, il faut supposer une nouvelle révolution agraire après 463/62 d'après le récit de Diodore. Ainsi :

« Après ces événements, ceux qui, au temps de la tyrannie de Hiéron, avaient été expulsés de leurs villes, revinrent dans leurs patries avec l'aide de leurs alliés et ils en chassèrent ceux qui s'étaient injustement emparés de villes qui appartenaient à autrui ; de ce nombre étaient les habitants de Géla, d'Agrigente et d'Himère. [...]Après cela, les habitants de Géla, qui avaient peuplé à l'origine Camarine, partagèrent le territoire de cette ville en lots ; presque toutes les villes s'empressèrent de mettre fin aux hostilités et prirent une décision commune : elles traitèrent avec les mercenaires étrangers installés dans le pays, accueillirent les exilés et rendirent leurs villes aux anciens citoyens cependant qu'elles permirent aux mercenaires, que les régimes tyranniques avaient mis en possession de villes qui ne leur appartenaient pas, d'emporter leurs biens et de s'installer dans le territoire de Messine. Donc les séditions et les troubles dans ces villes de Sicile cessèrent de cette façon et, ayant aboli les régimes politiques imposés par la domination étrangère, les cités partagèrent presque toutes leurs territoires respectifs entre tous les citoyens. » (II.76)

Cette situation idyllique, surestimée par Diodore, peut apparaître comme le fruit d'un compromis face au mouvement montant de Doukétios. Elle ne dure pas puisque à peine dix ans plus tard, en 454/53, la stasis s'impose de nouveau dans les cités, en particulier à Syracuse:

« On avait procédé dans les villes de Sicile à des inscriptions sur les listes civiques et à la redistribution des terres, mais beaucoup avaient été inscrits sans aucune règle et selon le hasard : aussi les villes furent-elles en proie à la fièvre et tombèrent-elles à nouveau dans les discordes civiles et les troubles ». (1 1.86$)$

S'il est difficile de faire la part des situations spécifiques à chaque cité dans ce tableau optimiste et globalisant, on peut s'interroger toutefois sur la prospérité de la Sicile vers le milieu du $\mathrm{V}^{e}$ siècle. Là aussi, sur le terrain, la situation n'a pas dû être aussi linéaire qu'il n'y paraît et l'exploitation des domaines a dû connaître des variations. Si l'on compare le texte diodoréen à celui de Lysias au sujet de la situation agricole de l'Attique au début du IV $\mathrm{V}^{\mathrm{e}}$ iècle ${ }^{66}$, on voit bien que les différentes crises qui ont affecté

65 Voir l'exemple d'Halaisa et des Tables d'Halaisa qui ont pu être interprétées comme le produit d'une redistribution de terres : Prestianni Giallombardo 1998.

66 Surl'olivier.

DHA supplément 6 
la cité (guerre du Péloponnèse, gouvernement des Trente, rétablissement par à-coups de la démocratie à la fin du $\mathrm{V}^{\mathrm{e}}$ siècle) ont mené à un turn-over de la propriété agraire et considérablement appauvri les campagnes. Il devait en être de même en Sicile où les bouleversements politiques ont dû avoir des conséquences sur la prospérité agricole. Le temps de la terre impose un rythme spécifique à ses occupants, fondé sur la longue durée et la stabilité, surtout dans le cas des cultures arbustives. Il faut donc probablement nuancer le tableau de la prospérité des campagnes à deux niveaux. D'abord, en envisageant au moins trois étapes de leur exploitation : le temps de la tyrannie où la stabilité du régime a offert aux exploitants la paix nécessaire à la productivité et à la rentabilité de leur propriété ${ }^{67}$; le temps des troubles civiques et extérieurs (avec le mouvement de Doukétios) qui a dû voir outre les destructions, des abandons de bien-fonds, vraisemblablement autour du milieu du $\mathrm{V}^{\mathrm{e}}$ siècle ; puis dans les quatre dernières décennies du $V^{e}$ siècle où la situation politique interne semble stabilisée, une reprise de la production agraire, mais qui n'a dû porter ses fruits qu'au moment de l'invasion carthaginoise, d'où la description de Diodore. Ensuite en distinguant les différentes régions de la Sicile dont les vicissitudes historiques varient d'une cité à l'autre, comme on le soulignera dans le peuplement du territoire. La situation de Sélinonte, demeurée à l'écart des grands mouvements de la Sicile sud-orientale, ne peut donc être identique à celle de Syracuse ou de la plaine de Catane et le texte de Diodore gomme les différences locales.

En outre, le texte de Diodore sur la révolution syracusaine associe toujours citoyenneté/ attribution des charges publiques et distribution de terres (II.72-73), ce qui me paraît peut-être un indice dans une autre quaestio vexata : la répartition de la population entre ville et campagne. L'historien insiste sur le fait que les citoyens promus par les tyrans refusent de se voir refuser leurs droits politiques au retour du gouvernement démocratique et menacent le nouveau régime : «Écartés de l'honneur conféré par l'élection aux magistratures, ces hommes supportaient difficilement cela et, de concert, ils se révoltèrent contre les Syracusains ». Or pour exercer sa citoyenneté dans une cité grecque, il faut être au centre du pouvoir politique, c'est-à-dire en ville, en ce qui concerne la Sicile. Même à Athènes, où l'on est sûr de la coexistence de structures politiques dans la chôra, le lieu où il faut être, c'est l'asty. Ce qui, à mon avis, suggère aussi que les citoyens influents résidaient en ville et non pas sur leurs terres.

En ce qui concerne le peuplement des territoires, Diodore offre une vision très déshumanisée des campagnes siciliennes. On lit la présence de phrouria et de poleis, mais

67 Diodore, II.38.I : « Gélon gouvernait avec sagesse les Sikéliotes, assurant aux villes en même temps qu'une excellente administration une grande abondance de tous les biens nécessaires à la vie ». 
les termes généralement utilisés pour désigner l'exploitation agricole ${ }^{68}$ n'apparaissent pas chez Diodore, sinon celui d'épaulis, qui évoque l'idée d'un gîte au plein air et qui est attesté dans les passages siciliens traitant de la fin de l'époque hellénistique. L'historien emploie généralement ce terme lorsqu'il parle de l'Orient plus ou moins mythique, ou de la Grèce égéenne du $V^{e}$ siècle dans le cadre de la guerre du Péloponnèse : on voit en effet les troupes des deux camps ravager le territoire et les épauleis ${ }^{6}$. La question du peuplement agraire et de la répartition de la population entre ville et territoire a été l'une des thématiques les plus discutées en démographie historique du monde grec antique et a été reprise récemment à la suite de l'énorme travail réalisé par l'équipe de M.H. Hansen ${ }^{70}$ et développée dans un travail de synthèse en $2006^{71}$.

Selon M.H. Hansen, dans une démonstration que l'on ne peut accepter dans sa totalité, l'archéologie montre de plus en plus que la majorité de la population vit en ville à l'époque classique. La vieille théorie selon laquelle la ville serait essentiellement un centre de consommation, mais où la majorité de la population vivrait dans le territoire, n'est pas opérante. S'appuyant sur les résultats de prospections dans un certain nombre de régions de la Grèce égéenne, mais également sur l'exemple de Métaponte où les calculs les plus raisonnables ont évalué la population de la ville à un ratio de 2 :I par rapport à celle de la campagne ${ }^{72}$, M.H. Hansen réévalue le modèle de l' « Ackerbürger » weberien, agriculteur citadin, qui se déplacerait depuis son domicile urbain jusqu’à son champ. Les consommateurs urbains seraient donc les producteurs eux-mêmes ${ }^{73}$.

68 On ne connaît pas de nomenclature unique pour désigner la ferme en grec ancien : Cf. Casevitz 1986. On trouve les termes de époikion (Chantraine 1999, s.v. Oikos), celui de agros (Chantraine 1999, s.v. agros), de gèdion (Chantraine 1999, s.v. Gè) : celui de Kleision (Chantraine 1999, s.v. klinô). Le terme de purgos qui a donné lieu à une abondante littérature sur les tours de campagne n'est pas attesté en ce sens pour l'antiquité (Liddell-Scott, s.v. Purgos ; Chantraine, s.v. purgos). Il semble que le terme de maison oikos et ses dérivés aient suffi à la désigner lorsque les sources ne se contentent pas d'évoquer ceux qui habitent la campagne : cf Gallo 2001, en particulier p. 62-63.

69 Mc Dougall I983, s.v. epaulis.

70 Hansen et Nielsen 2004.

71 Hansen 2006a.

72 J.C. Carter (2006) a évalué le territoire de Métaponte à environ $200 \mathrm{~km}^{2}$ et le nombre de fermes à 870 entre 350 et 300 (ce qui chiffrerait la population de la campagne à 4.500/9.000 personnes (selon que l'on compte 5 à Io personnes par ferme). À partir d'un calcul maximal de IO4 îlots de 24 maisons en ville, il a proposé un chiffre de 2.500 maisons à la même époque, ce qui correspondrait à une population urbaine maximale d'env. 12.500 à 25.000 habitants. Mais d'après les photographies aériennes, il faudrait plutôt compter 75 îlots de 20 maisons (Muggia 1997). Au total on aurait donc un nombre total de 1500 maisons, soit près du double des exploitations familiales en campagne.

73 Hansen 2004.

DHA supplément 6 
À l'autre extrémité du monde grec, E. Greco soutient la même hypothèse pour la Grande Grèce ${ }^{74}$. Constatant que les premiers établissements d'époque archaïque étaient situés à une dizaine de kilomètres de la ville minimum, il applique les modèles explicatifs conçus pour la Grèce métropolitaine aux territoires connus de Métaponte, Sybaris ou Tarente. Les agriculteurs auraient habité en ville, mais seraient allés travailler dans les champs à une distance qui pouvait se parcourir dans la journée, soit à $5 / 7 \mathrm{~km}$ du centre urbain. Comme partout en Méditerranée, l'époque classique verrait une mutation profonde de ce peuplement, à savoir une véritable explosion des établissements dans le territoire. Il s'appuie non pas tant sur l'exemple de Métaponte que sur celui de Thourioi (en particulier l'édifice $\mathrm{C}$ du quartier de Stombi et les sources littéraires concernant la colonie panhellénique), de Vélia et bien sûr les tables d'Héraclées. L. Gallo, s'appuyant sur les recherches antérieures, défend la même hypothèse pour la Sicile (I989).

Les deux thèses sont acceptables en fonction des exemples que l'on choisit, et c'est là que réside la principale difficulté. Nous sommes à ce point tributaires de notre documentation qu'il faut, en particulier lorsqu'elle est aussi lacunaire, garder un maximum de prudence. À vouloir modéliser, on risque de passer à côté de ce qu'était la réalité d'une occupation rurale, probablement plus discontinue et chaotique que ne le souhaiterait l'historien. Si l'on regarde la situation insulaire, les résultats siciliens peuvent-ils contribuer au débat ? Nous disposons d'un côté du témoignage de Diodore, de l'autre d'une documentation archéologique qui varie considérablement d'une région à l'autre.

Dans l'ensemble, en Sicile, le nombre de fermes répertoriées pour le $V^{\mathrm{e}}$ siècle est faible. Ainsi dans le catalogue dressé en 1994, on ne trouve qu'une quinzaine de sites occupés à l'époque classique, et le plus souvent au $\mathrm{IV}^{\mathrm{e}}$ siècle, sur un total plus d'une centaine d'exploitations pour les époques hellénistique et romaine ${ }^{75}$. L'essor du peuplement des campagnes se poursuit aux époques hellénistique et romaine, comme l'atteste alors la multiplication des fermes permanentes ${ }^{76}$. Ce qui signifie que la plupart de nos témoignages sont postérieurs au raid carthaginois de 409-405. Ce faible chiffre tient bien sûr d'abord à l'état de la recherche sicilienne, puisque rares sont encore les territoires prospectés. Mais même dans les zones passées au crible du survey, les résultats sont médiocres. Ainsi, dans le territoire d'Agrigente, on a répertorié plusieurs fermes

74 Greco 1999b; Greco 200I.

75 Bove 1994.

76 Voir par exemple dans le territoire de Ségeste qui a fait l'objet d'intenses prospections, De Angelis 2000, p. 195. L'essor de la ville hellénistique va de pair avec une forte occupation des campagnes environnantes. 
répertoriées à Palma di Montechiaro ${ }^{77}$, à Sciacca ${ }^{78}$, à Grancifone ${ }^{79}$, mais aucune dans la campagne immédiate de la ville grecque. Dans le territoire de Géla, les découvertes sont datables surtout à partir du $\mathrm{IV}^{\mathrm{e}}$ siècle $^{80}$. Là aussi, les établissements répertoriés sont à une distance respectable du centre urbain. Plus à l'ouest, dans le territoire d'Héracléa Minoa, les investigations menées par R.J.A. Wilson, n'ont révélé aucune exploitation d'époque archaïque et classique ${ }^{81}$. Le territoire de Sélinonte est pratiquement inconnu $^{82}$. Dans la zone d'épicratie syracusaine, les exploitations connues sont encore rares : une ferme, occupée entre le $\mathrm{VI}^{\mathrm{c}}$ et le $\mathrm{IV}^{\mathrm{e}}$ siècle, entre Noto et Rosolini qui appartenait vraisemblablement au territoire d'Héloros ${ }^{8_{3}}$, quelques indices dans la zone de Cassibile à moins de dix kilomètres de Syracuse ${ }^{84}$ et des découvertes récentes qui n'ont pas encore fait l'objet de publications.

Dans le territoire de Camarina ${ }^{85}$, on connaît un certain nombre d'exploitations agraires : à immédiate proximité de la ville, dans la limite des $5 / 7 \mathrm{~km}$, la 'fattoria Jurato', la 'fattoria Capodicasa,' la 'fattoria Piliria' et la 'fattoria delle api' ${ }^{86}$, tandis que les autres exploitations connues sont situées en dehors du périmètre de marche quotidienne, comme la 'fattoria Menta ou la 'fattoria Cancellieri'. S'appuyant sur la documentation littéraire, et observant des orientations identiques intra et extra muros, les fouilleurs y ont vu la preuve d'une réorganisation de l'espace civique à l'occasion de la refondation démocratique de 466 mentionnée par Thucydide (6.5.3) et Diodore de Sicile (supra): les habitants de Géla en tant que refondateurs de la cité auraient opéré une nouvelle cadastration en klèroi distribués notamment à ceux qui avaient été chassés et déchus de leurs droits dans le régime précédent. Ce parcellaire serait décelable sur le

77 Castellana 1983.

78 Gaddimi : Mc Connell, 1989 ; contrada Guardabasso : Mc Connell 1990 ; contrada Locogrande : Bejor 1975, p. 1294-95; contrada Montagnola : Giustolisi 1981, p. I19.

79 Castellana 1990. La ferme remaniée au IV ${ }^{\mathrm{e}}$ siècle est constituée d'une cour bordée au nord par trois pièces dont l'une comportait un pressoir à huile, l'une un pressoir à vin et la troisième semblait destinée au stockage des denrées.

80 Contrada Ficuzza : Canzanella 1989, p. 456 ; et Contrada Gurgazzi : Canzanella 1990 ; contrada Priorato, Adamesteanu 1958, Manfria, Milingiana Sottana, Adamesteanu 1958 ; Monte Desusino-Butera Panvini 1993-94.

8I Wilson 1980. Les exploitations agraires de Campanaio et de Castagna, bien connues par les fouilles de R.J. Wilson, ne sont pas antérieures à l'époque hellénistique : Wilson 1994.

82 Bianchi et al. 1998.

83 Guzzardi 200I.

84 Turco 1990.

85 En dernier lieu Di Stefano G. 200 I.

86 Di Stefano et Anelli 20oI.

DHA supplément 6 
terrain grâce à la présence de tombes, de canaux, de murets et de fermes qui adopteraient la même orientation que l'urbanisme de la ville elle-même. L'hypothèse est séduisante, mais les indices sont, à mon avis, trop ténus pour fixer une datation aussi définitive : les quelques fermes repérées et partiellement fouillées présentent une phase d'occupation principale des époques postérieures, même si on y a découvert quelques indices du $V^{e}$ siècle. Il est difficile en outre de ne pas tenir compte du passage des Carthaginois en 405 qui s'emparent de la ville et doivent ravager le territoire comme le font toujours les armées lancées sur une cité. La situation camarinéenne est, à mon avis, d'autant plus complexe que la cité a représenté un enjeu important dans les relations entre Géloens et Syracusains, suscitant des conflits destructeurs menant à plusieurs refondations et réinstallations de populations de l'une ou l'autre des deux métropoles. Sans adopter l'interprétation proposée par G. Di Stefano, trop exemplaire selon moi pour être vraie, on doit reconnaitre qu' il existe des fermes dans le périmètre immédiat du centre urbain, accréditant la thèse défendue par E. Greco pour la Grande Grèce.

Les tombes ou nécropoles attestent également une occupation durable du territoire. À Agrigente, c'est le cas de la nécropole Mosè, située à quelques kilomètres à l'est de la cité. Ouverte, semble-t-il, à la fin du VI e siècle, elle est occupée pendant tout le $\mathrm{V}^{\mathrm{e}}$ siècle par des monuments dont le faste induit un certain coût de la construction et suggèrent ainsi une aisance réelle de leurs propriétaires ${ }^{87}$. Dans ce cas précis, la présence d'une véritable nécropole entérine plutôt la présence d'un village que de propriétés isolées. À Géla, les nécropoles Contrada Spina Santa et Farello ${ }^{88}$, situées à l'est de la ville, montrent une occupation du site pendant les trois premiers quarts du $\mathrm{V}^{\mathrm{e}}$ avec une interruption à la fin du siècle qui doit correspondre aux destructions opérées par les Carthaginois en 405. On les a interprétées comme appartenant à une exploitation ou un hameau agricole, mais la colline de Géla étant d'une superficie limitée ${ }^{89}$, elles ont pu abriter les dépouilles d'habitants de faubourgs, comme c'est le cas à Himère aujourd'hui mieux connu par les fouilles de S. Vassallo et N. Allegro. Seule la dispersion de tombes dans la plaine peut conforter la thèse d'un peuplement fixe de la campagne ${ }^{90}$.

Si l'on reprend l'exemple le plus étudié des sites sikéliotes, à savoir la chôra d'Himère, les prospections territoriales des Palermitains ${ }^{91}$ ont identifié peu de sites à proximité de la cité grecque pour l'époque archaïque. La mutation agraire qu'ils

87 De Miro 1988, p. 244-248.

88 Giudice et al. 2003, p. 48 avec bibliographie antérieure.

89 La superficie de la ville archaïque et classique est estimée à ıos ha.

90 Belvedere 200I, p. 720-72I.

91 Alliata et Belvedere 1988 ; Belvedere 200I. 
observent pour l'époque classique, à savoir l'usage intensif des sols, entraîne une diffusion de l'occupation rurale entre le fleuve Himéras et le fleuve Roccella à l'est, entre l'Himéras et le Torto à l'ouest. Cette multiplication des sites dénote, selon les fouilleurs, l'émergence d'une petite propriété foncière à gestion familiale, mais ne s'étend pas au-delà d'une ligne située à $9 \mathrm{~km}$ de profondeur et à $8 \mathrm{~km}$ environ de largeur et réserve une zone libre de tout établissement stable dans un diamètre de $3-4 \mathrm{~km}$ autour de la ville antique.

Dans certaines zones de l'île, en revanche, on assiste à une rétractation de l'habitat territorial comme dans la zone élyme où des sites prospères au milieu du $\mathrm{VI}^{\mathrm{e}}$ siècle disparaissent au plus tard vers 475. I. Morris et $S$. Tusa ont proposé de manière convaincante d'interpréter cette situation comme un regroupement progressif des populations locales dans les grands centres urbains, tels Ségeste, Entella, Erice, ou Halikyai (connue pour l'instant uniquement par les sources). Pour eux, il ne s'agit nullement d'un déclin démographique et économique de ces populations ${ }^{92}$, mais d'une réorganisation du territoire autour d'une centralisation urbaine. On sait qu'à Syracuse, le synœcisme décrété par Gélon entraîna le dépeuplement ${ }^{93}$ de certaines cités prospères au $\mathrm{VI}^{\mathrm{e}}$ siècle, comme Camarina, Mégara Hyblaea ou Léontinoi, pour les plus célèbres. Curieusement d'ailleurs, Diodore ne dit rien de ce synœcisme. Dans un contexte politique de constitution des éparchies agrigentine et syracusaine, il est probable qu'un tel regroupement ait pu s'appliquer aux villes sikéliotes en général, où les conditions de confort se développent au cours du $\mathrm{V}^{\mathrm{e}}$ siècle, offrant ainsi un cadre de vie plus agréable aux habitants. À Agrigente comme à Syracuse, la mise en place d'un réseau d'aqueducs offrait aux citadins des ressources en eau faciles et salubres de même que la construction d'aménagements publics et religieux facilitait la cohésion du corps civique et la pratique religieuse. La pression démographique dont parlent les sources littéraires, même s'il faut réévaluer les chiffres donnés ${ }^{94}$, est réelle dans toutes les cités, probablement aussi dans le contexte de paix générale décrit par Diodore. L'historien estime à vingt mille citoyens, chiffre attribué à Timée de Tauroménion's, et deux cent mille habitants la seule ville d'Agrigente. On a pu contester ces chiffres mais ils ne paraissent pas si démesurés si l'on se fonde sur les chiffres proposés pour Athènes en 43I (30.000 citoyens et 200.000 habitants avec les résidents étrangers ${ }^{96}$, qu' il faut probablement assimiler aux métèques

\footnotetext{
92 Morris et Tusa 2004.

93 Hérodote parle de destruction : 7. 156.

94 De Waele 1980.

$95 \mathrm{FrGH} 566 \mathrm{~F}_{2} 6.84 .3$ Jacoby.

96 En dernier lieu Hansen 2006b.
}

DHA supplément 6 
athéniens) et sur l'ampleur du territoire agrigentin ${ }^{97}$. La description d'Agrigente, de Sélinonte et d'Himère au moment des sièges de 409-405 montre qu'il s'agissait de cités populeuses et cosmopolites ${ }^{98}$.

Dans le débat qui concerne les rapports peuplement agraire/peuplement urbain, il faut donc là aussi rester prudent, les situations pouvant différer d'une cité à l'autre. Il est probable que dans le territoire des grandes cités, le peuplement se soit disséminé, mais il faut peut-être le mettre en lien avec la vie politique et la composition sociale des cités que j'évoquais supra. La citoyenneté exigeant la présence dans le centre urbain, les fermes identifiées ici ou là peuvent très bien être la résidence des exploitants de la propriété, pas nécessairement des propriétaires et prend ici tout son sens la thématique de l'esclavage indigène et domestique, dont on a du mal à saisir les contours en l'absence de sources textuelles sur le sujet. D'autant que, même à des époques plus reculées, la nécessité d'une main d'œuvre agraire impose une présence dans la campagne plus constante qu'on ne le croit.

Le témoignage de Diodore permet d'ailleurs de mieux connaître les villes que leurs campagnes, notamment la société des grandes poleis classiques, au moins celles d'Agrigente et de Syracuse (éclairée surtout par les troubles politiques qui l'agitent). Si l'on prend l'exemple d'Agrigente, Diodore nous offre la vision d'une élite économique et politique dont l'éducation et les mœurs ne doivent rien aux meilleures sociétés métropolitaines et où les pratiques funéraires témoignent du luxe et de la tryphè des élites agrigentines 99 . La description du mode de vie des Agrigentins est unique chez Diodore qui dit l'emprunter à Timée, Empédocle et Polyclite de Larissa (13.82-84). Il cite ainsi l'existence de tombes érigées à des chevaux ou aux oiseaux des filles et jeunes garçons. Au-delà de l'exagération probable que l'on a interprétée comme la marque du pythagoricien Timée, la cavalerie est le point fort des armées grecques de Sicile et l'élevage de chevaux est un des aspects majeurs de l'économie insulaire, si l'on en juge notamment par la référence aux vainqueurs des Jeux Panhelléniques, essentiellement dans les courses de chars. D'après les épinicies de Pindare, la majorité des vainqueurs aux courses de chevaux ou de mules sont des Siciliens. Diodore lui-même souligne le

97 Il est estimé à la suite de l'Inventory of Archaic and Classic Poleis à plus de $500 \mathrm{~km}^{2}$ (Hansen 2006a, appendix 4. p. I07-116).

98 La bibliographie sur la question est abondante : cf. par ex. Gallo 1989 et l'état de la bibliographie ; pour Agrigente, De Waele 1980 ; pour Himère, Asheri 1971. À Himère, aux chiffres proposés par Asheri en 197I ( 10.000 habitants sur le plateau et I0.000 dans le territoire), il faut ajouter la population de la ville basse en cours d'investigation. On a ainsi proposé une population totale de 16.000 à 18.000 habitants (en comptant une moyenne de 1500 à 2000 âmes par ha et une superficie totale de $80 \mathrm{ha}$ ).

99 Collin Bouffier 1999. 
sens de l'hospitalité de l'Agrigentin Gellias-Tellias, celui-ci accueillant cinq cents cavaliers sous son toit ou la richesse de son compatriote Antisthénès qui fait suivre le char nuptial de sa fille de huit cents cavaliers. Les sépultures de chevaux, qui peuvent apparaître comme un témoignage de tryphè, peuvent aussi être vues comme une spécificité des élites sikéliotes accordant à leurs meilleurs chevaux, vainqueurs dans les concours grecs, les honneurs qu'elles estiment leur devoir. Certains des auriges se font même enterrer avec le prix de leur victoire, comme en témoigne la présence d'une amphore panathénaïque dans une tombe agrigentine des environs de 500 décorée d'un quadrige en course ${ }^{100}$. L'archéologie funéraire a mis en relief la richesse de la population agrigentine au $V^{e}$ siècle ${ }^{101}$. Diodore, toutefois, ne porte aucun jugement, même lorsqu'il évoque les restrictions au mode de vie des Agrigentins qui, à la veille de l'assaut carthaginois de 406/405, n'ont le droit de monter la garde qu'avec un tapis, un matelas, une couverture et deux oreillers! Contrairement à Timée qui a dû constituer l'essentiel de sa source, il se contente de décrire une sorte d'âge d'or auquel la guerre carthaginoise met un terme définitif. On a changé d'époque.

\section{Un âge d'or culturel}

C'est probablement dans ce champ qu'est le plus perceptible l'âge d'or décrit par Diodore. L'historien s'attarde longuement sur les travaux réalisés par certaines des cités pour embellir leurs villes et améliorer le confort de leurs citoyens ${ }^{102}$. Selon lui, la bataille d'Himère a ouvert l'ère des constructions, grâce au butin et aux prisonniers carthaginois. Mais la prospérité économique a tout autant permis l'essor architectural et artistique des villes.

« Les cités enchaînèrent les prisonniers qu'elles avaient reçus et les employèrent aux travaux publics. Les Agrigentins qui en avaient reçu le plus grand nombre, embellirent leur ville et leur territoire ; il y avait chez eux une telle quantité de captifs que nombre de particuliers en possédaient cinq cents. Ce qui leur procura cette masse de prisonniers, c'est non seulement que les Carthaginois avaient envoyé beaucoup de soldats à la bataille, mais aussi qu'après la déroute, beaucoup parmi les fuyards avaient gagné l'intérieur des terres et tout particulièrement le territoire

I00 Veder greco 1988, n$^{\circ} 60$, p. 196.

Iо I Cf. Veder greco 1988, cité supra. On rétorquera que la présence de mobilier funéraire n'est pas nécessairement synonyme de richesse, comme l'a bien montré I. Morris dans les années 80 (Morris 1987), mais dans le cas d'Agrigente, les différences entre des tombes de même époque semblent bien correspondre à des statuts socio-économiques variés.

I02 Pour un bilan, voir Blandini 1999. On pourra consulter Van Compernolle Th. 1992, commode mais aujourd'hui vieilli.

DHA supplément 6 
d'Agrigente et comme ils avaient été tous été capturés vivants par les Agrigentins, la ville se trouvait remplie de captifs. La plupart furent employés au service de la collectivité, les uns taillèrent les pierres qui servirent non seulement à la construction des très grands temples en l'honneur des dieux, mais aussi à celle de canalisations souterraines pour l'écoulement des eaux hors de la ville, grandiose réalisation qui mérite d'être vue bien qu'on puisse les négliger pour les faibles dépenses engagées $^{103}$. 》

Agrigente attire de nouveau l'attention de l'historien qui ne consacre guère de place à Syracuse et on peut s'en demander les raisons, vu que la présentation même de Gélon est très laudative et qu'il aurait été logique d'y ajouter sa politique de constructions ${ }^{104}$. La Sicile apparaît comme une sorte de paradis, dont la kolymbethra est le parangon. Les constructions se distinguent soit par leur aspect démesuré, notables aux dépenses somptuaires des édifices religieux, soit par leur originalité (en ce qui concerne les travaux hydrauliques d'Agrigente, sans parallèle avant l'époque hellénistique dans le monde grec). Comme l'avait bien étudié R. Martin ${ }^{105}$, les dépenses occasionnées par les constructions civiques des Sikéliotes aux $\mathrm{VI}^{\mathrm{e}}$ et $\mathrm{V}^{\mathrm{e}}$ siècles sont extrêmement lourdes. Estimant le financement des édifices religieux à partir des cubages de pierre nécessaires à la construction, il évaluait le coût de quatre des temples agrigentins du $\mathrm{V}^{\mathrm{e}}$ siècle, le temple dit de la Concorde à 54 talents, le temple dit d'Héra Lacinia à 78 talents, le temple dit d'Héraclès à 216 talents et l'Olympieion ${ }^{106}$, demeuré inachevé, à 1512 talents, soit un total de 1864 talents. En comparaison, les dépenses de l'Acropole d'Athènes, pourtant considérables pour les autorités athéniennes, apparaissent comme dérisoires puisque le Parthénon a été estimé entre 470 et 500 talents, les Propylées à 200. Outre le temple de Zeus, seul mentionné par Diodore, les édifices construits pendant le $\mathrm{V}^{\mathrm{e}}$ siècle sont au moins au nombre de quatre, si on se limite aux seuls temples d'envergure, le temple dit d'Héraklès ${ }^{107}$, le temple dit de Vulcain ${ }^{108}$, le temple dit de la Concorde, le temple dit de Junon, et que l'on néglige les nombreux édifices du sanctuaire occidental

I03 Traduction Haillet remaniée.

I04 Mafodda 1996.

I05 Martin 1987, p. 533-547.

IO6 Je maintiens la datation traditionnelle du temple de Zeus Olympien. On a contesté dans un certain nombre d'articles le lien entre Himère et la construction de l'Olympieion : selon leurs auteurs, le temple aurait été commencé par Théron avant la bataille de 480 . En parallèle avec les cités d'Athènes ou de Sélinonte, le choix iconographique de la Gigantomachie a été interprété comme une marque de la victoire de l'Emménide sur ses opposants et le signe d'une politique de propagande anti-aristocratique : voir par exemple, Barbanera 1995, p. 89-96. Mais les arguments avancés n'emportent pas l'adhésion face aux témoignages antiques eux-mêmes et aux conditions socio-économiques nécessaires à la construction.

107 En dernier lieu Mertens 2006, p. 315.

I08 Zoppi 1999. 
de la terrasse des temples ${ }^{109}$ ou le temple des divinités chthoniennes à l'extrémité orientale de la Rupe Atenea.

Le temple de Zeus est de loin le plus somptueux puisqu'il figure parmi les exemples du gigantisme de l'architecture grecque, je ne reprendrai pas ce que tous connaissent $^{110}$. Diodore décrit certaines de ses caractéristiques, en particulier la présence des colonnes anthropomorphes et le décor iconographique des frontons ${ }^{111}$ : une Gigantomachie à l'est et une Ilioupersis à l'ouest, dont certains fragments ont pu être identifiés. On a longtemps débattu de cet édifice atypique dont ni la datation, ni la typologie ne trouvent encore de consensus. La plupart des interprétations convergent vers une datation post-himéréenne par analogie avec la politique de Gélon, qui, de son côté, consacre à Olympie le trésor des Syracusains chargé d'abriter une statue de Zeus colossale et les trois tuniques de lin qu'il lui offre (Paus. 6.19.7), tandis qu'à Syracuse, il habille la statue de culte de l'Olympieion d'un manteau d'or. Dans chacune des épicraties siciliennes, le même hommage serait rendu au souverain de l'Olympe, de manière tout à fait conforme à ce que l'on observe dans les cités de la Grèce égéenne. Le Zeus d'Olympie est le dispensateur des victoires guerrières, le garant des accords internationaux et des traités de paix.

Mais la politique de grands travaux réalisés à Agrigente et peut-être à Syracuse, dans le champ de l'architecture religieuse et hydraulique, me semble témoigner également d'une influence des modèles perses de la dynastie achéménide. Un faisceau d'indices nous incite en effet à mettre l'accent sur les liens au moins culturels, qui ont pu se tisser entre les deux régimes. Comme on le voit très bien chez Hérodote ${ }^{112}$, les Perses symbolisaient le luxe et de la richesse au début $\mathrm{du}^{\mathrm{e}}$ siècle ; ils ont pu apparaître comme des exemples à suivre pour les régimes autocratiques. On l'a déjà proposé pour les tyrannies égéennes de la fin du VI ${ }^{\mathrm{e}}$ siècle. À Agrigente, le parallèle a été dressé par C. Marconi sur l'emploi de figures masculines pour porter l'architrave et la toiture du temple de Zeus. Interprétées soit comme des atlantes, soit plus pertinemment comme des Titans ${ }^{113}$, par parallèle avec le culte de Zeus et la victoire de Grecs contre des barbares, ces figures rappellent une iconographie analogue dans l'empire achéménide : celle des peuples vaincus dans les villes du Grand Roi, en particulier à Persépolis sur la porte Est du Tripylon. De même, la kolymbethra m'était apparue comme un exemple

\footnotetext{
I09 De Miro 2000.

IIO De Waele 1989; Mertens 2006, p. 26I-66.

iI De Waele 1982.

II2 Dans son récit de la bataille de Platées en particulier : Hdt 9.80.

II3 Marconi 1997.
}

DHA supplément 6 
des liens au moins culturels entre la tyrannie emménide et l'empire du grand $\operatorname{Roi}^{114}$. Je ne reprendrai pas non plus ici le dossier. Je me contenterai d'insister sur l'influence qu'ont pu avoir les réalisations achéménides sur les tyrans d'Agrigente. Selon Diodore,

« Les Agrigentins réalisèrent aussi une kolymbethra somptueuse d'un périmètre de sept stades [entre $\mathrm{I} 200$ et $\mathrm{I} 400 \mathrm{~m}$ ] et d'une profondeur de vingt coudées [soit neuf mètres]. Lorsqu'ils y eurent amené l'eau de fleuves et de fontaines, elle devint un vivier piscicole, qui fournit de nombreux poissons pour l'alimentation et le plaisir des Agrigentins; et lorsqu'une très grande quantité de cygnes s'y installa, sa vue devint charmante » (II.25)

« Et il y avait aussi un lac artificiel, creusé à l'extérieur de la cité, d'un périmètre de sept stades et d'une profondeur de vingt coudées ; les Agrigentins, après y avoir amené l'eau, aimaient à y produire une quantité de poissons de toutes sortes en vue des banquets publics, avec lesquels cohabitaient des cygnes et une multitude d'autres oiseaux ; aussi ce spectacle offrait-il un grand spectacle aux spectateurs. » (I3.82)

J'avais écarté il y a quelques années certains aspects de la description diodoréenne, notamment la présence d'un vivier piscicole aussi vaste à une telle distance de la mer, alors que les Grecs préfèrent les poissons de mer aux poissons d'eau douce. Il n'en reste pas moins que cet aménagement apparaît comme un unicum dans le monde grec, d'où l'insistance de Diodore à son sujet ${ }^{115}$. Le bassin artificiel avait pour objectif principal de constituer une réserve d'eau sur un site assez défavorisé de ce point de vue : la pluviométrie agrigentine est comparable à celle de Carthage et la géomorphologie calcaire entraîne les précipitations directement en profondeur et vers la plaine côtière. L'aspect exceptionnel du projet doit, à mon avis, être mis en relation avec ce qui se produisait en Asie Mineure depuis la deuxième moitié du VI siècle. En Perse le souverain crée, dans les villes où il réside, des paradis concentrant l'ensemble de la flore et de la faune de ses possessions ${ }^{116}$, aussi bien à Sardes qu'à Persépolis; en Sicile, un bassin dans lequel se déplacent des cygnes et autres oiseaux, et où l'on peut pêcher toutes sortes de poissons, serait une pâle copie d'un modèle impossible à imiter dans sa totalité en Occident. De même, un fragment de Diodore, rapporté par Athénée de Naucratis, évoque la création de deux paradis (c'est le terme employé), l'un à Syracuse par Hiéron I ${ }^{\mathrm{er}}$, l'autre à Rhégion, probablement par Anaxilas, qui n'ont pas été identifiés ni localisés (Ath., I2, 542a). Or à Syracuse, il existe un quartier dit 'du Paradis' à l'est du théâtre, où on a découvert, dès la fin du XIX ${ }^{\mathrm{e}}$ siècle, les vestiges d'un aqueduc ${ }^{117}$. On

II4 Collin Bouffier 2000.

IIs Collin Bouffier 2000.

II6 Briant 1996, p. 94-96; 213-216.

II7 En dernier lieu Messina 2009; Guzzardi 2000. 
ne sait pas actuellement à quelle époque remonte la dénomination du quartier mais il serait intéressant de confronter toute la documentation sur le sujet. De toutes façons, toute la zone est intéressée par la politique urbaine des Deinoménides et il est suggestif d'envisager à proximité du théâtre, l'existence d'un jardin comparable à ce que devaient être les environs de la kolymbethra. Si l'on collecte ainsi les infimes références, le parallèle avec l'Orient, qui n'est plus phénicien cette fois, devient manifeste. On pourrait aller plus loin en suggérant l'instauration d'autres influences. Diodore évoque, lors du récit de la bataille d'Himère, les relations présumées par certaines de ses sources entre Carthaginois et Perses; mais les rapports entre Grecs de Sicile et Perses semblent bien avoir existé également. Ainsi la mise en place des royautés gélonienne et théronienne, avec les titulatures de 'Basileus', 'Sôter' et 'Euergétès', a quelque chose d'oriental que l'historien n'a peut-être pas perçu clairement. Comme on l'a vu supra, les trois termes ne figurent pas au répertoire politique de l'époque classique.

Quant au réseau d'huponomoi réalisés à Agrigente, que l'on traduit généralement par le terme d'égouts' ${ }^{118}$, on ne peut pas adhérer à cette interprétation lorsque l'on connaît le réseau dense de canalisations répertoriées sur l'ensemble du site ${ }^{119}$. Si certaines portent effectivement les eaux hors de la cité, la plupart sillonnent la zone urbaine pour alimenter les quartiers par de vraisemblables fontaines. On ne connaît pour l'instant que les fondations d'un réservoir, l'hypogée Giacatello, dont l'étude architecturale serait probablement très éclairante. Comme je le soulignais supra, les conditions hydrologiques d'Agrigente placent la cité en zone semi-aride, et l'urbanisation du site a rendu nécessaire une politique collective d'approvisionnement en eau, que l'on attribue la réalisation des travaux aux Emménides ou au régime qui leur a succédé. En revanche, l'évacuation des eaux sur le site ne pose pas de véritable problème, dans la mesure où l'on ne se situe pas dans une zone à drainer ou à bonifier, comme c'est le cas dans un certain nombre de sites sikéliotes. Diodore nous indique également l'intervention d'un certain Phaiax, sur le nom duquel on a longtemps glosé : « Le dénommé Phaiax fut l'épistate de ces travaux et la renommée de ces aménagements fut telle qu' ils furent appelés canalisations phéaciennes, de son nom à lui ${ }^{120}$. » Qu'il s'agisse de son titre ou de son nom, Diodore ne donne pas plus de précisions. Le terme d'épistate, comme on l'a souligné pour d'autres textes ${ }^{121}$, est appliqué aussi bien au magistrat responsable d'un ouvrage

II8 Notamment Haillet 200I.

119 Arnone 1991.

I2O II.25.3: Traduction personnelle.

I21 Hellmann 1999, nº (Délos), p. 39-44 (350 av. J.-C) et surtout Syracuse, nº 42, p. 100-101 pour l'inscription de l'Apollonion du VI' siècle. 
décrété par la cité qu'au maître d'œuvre de l'ensemble d'un programme architectural, sorte d'entrepreneur, chef du chantier de construction, voire à l'architecte et concepteur du projet. En l'absence de toute autre information, on ne tranchera pas, même s'il est plus vraisemblable d'y voir le magistrat responsable des travaux. S'il s'agissait de l'inventeur du réseau, ce serait un cas exceptionnel dans nos sources que la référence à un architecte d'ouvrages hydrauliques. Seul le nom d'Eupalinos de Samos, créateur de l'aqueduc samien de la deuxième moitié du $\mathrm{VI}^{\mathrm{e}}$ siècle est passé à la postérité ${ }^{122}$. On a bien montré que même pour les époques archaïque et classique, les références étaient rares $^{123}$. En outre, on connaît d'autres épistates chargés par leur cité de construire des édifices religieux en Sicile, comme Phalaris (qui en profite pour s'emparer du pouvoir à cette occasion) ou le syracusain Agathoclès ${ }^{124}$ à des époques proches de celles de Phaiax. Enfin, même son nom ressemble davantage à un sobriquet, construit par analogie avec le caractère souterrain et donc obscur des galeries hydrauliques, sobriquet comme on en connaît d'autres en Sicile ${ }^{125}$. En tout cas, les canalisations connues par l'archéologie agrigentine sont toutes souterraines, ce qui aurait tendance à confirmer leur datation haute plutôt que leur fonction d'égout. Aucun ouvrage d'art susceptible de renvoyer le dispositif à l'époque romaine n'est connu et même si toutes les canalisations ne peuvent remonter au $V^{e}$ siècle, il est vraisemblable que la plupart d'entre elles ont été réalisées avant la destruction carthaginoise de 405 qui ôte à la cité appauvrie et dépeuplée les capacités financières et les raisons de mettre en place de tels aménagements. Les galeries captaient l'eau enfermée entre le banc d'argile et le calcaire de surface par l'intermédiaire de cavités circulaires qui drainaient l'écoulement des parois avant qu'il ne fût canalisé dans des conduites, repérées dès le $\mathrm{XVII}^{\mathrm{e}}$ siècle par les voyageurs et encore connues sur certains tronçons aujourd' hui ${ }^{126}$.

Le bilan est plus difficile à dresser en ce qui concerne Syracuse car l'archéologie syracusaine est assez pauvre en vestiges $\mathrm{du} \mathrm{V}^{\mathrm{e}}$ siècle. Après la victoire d'Himère, nous dit Diodore, « du butin, il [Gélon] garda les plus belles prises, car il voulait orner de ces dépouilles les temples de Syracuse ; il en fit aussi clouer beaucoup aux murs des plus célèbres sanctuaires d'Himère et répartir le reste ainsi que les prisonniers entre ses alliés,

\footnotetext{
I22 Hdt. 3.60

I23 Hellmann 1999, loc. cit.

I24 Dans un fragment de la Bibliothèque Historique, cité note 2.

I25 On en trouve de très nombreux exemples dans Dubois 1989 (Sélinonte, ${ }^{\circ}$ 34, p. $44 ; 35$, p. $45 ; 36$, p. $47 ; 38$, p. 5 I etc.).

I26 Arnone 1991 ; Miccichè 1996 ; Collin Bouffier 200I. En particulier dans le sanctuaire rupestre, Siracusano 1983.
} 
proportionnellement au nombre de soldats qu'ils avaient fournis. » (II.25). De même, « Gélon utilisa le butin pour construire à Déméter et Coré des temples importants. Il fit faire un trépied d'or de seize talents et le consacra dans le sanctuaire de Delphes en offrande de reconnaissance à Apollon. Il entreprit plus tard la construction d'un temple à Déméter dans la région d'Aitna, $† . .$. , mais à vrai dire, il ne l'acheva pas, le destin ayant interrompu ses jours. » (II.26.7). Les informations de Diodore sont confirmées et complétées par d'autres textes ${ }^{127}$ en ce qui concerne les offrandes delphiques et olympiques. Pour les temples syracusains eux-mêmes on a douté des réalisations géloniennes. Ainsi, selon M. Gras ${ }^{128}$, il s'agissait davantage d'équiper les temples que d'en édifier, le verbe kataskeuazein ayant plutôt ce sens alors que le verbe oikodomein serait généralement utilisé par Diodore lorsqu'il s'agit de construction. Toutefois on sait que le vocabulaire utilisé par les auteurs anciens n'est pas aussi systématique et précis qu’on ne le souhaiterait, et il est difficile de penser que Gélon se soit contenté d'aménager des sanctuaires existants et non pas de créer une architecture digne de son pouvoir alors qu'il offrait à la ville une extension nouvelle. Mais du fait de l'occupation constante du site syracusain au cours de l'histoire, la réalité architecturale des édifices religieux nous échappe en très grande partie. En ce qui concerne l'architecture monumentale de prestige, seuls les temples d'Apollon et de Zeus Olympien sont assurément antérieurs et la datation du temple ionique, attribuée jusque là au $\mathrm{VI}^{e}$ siècle, est aujourd'hui remise en question par les dernières fouilles. Les privilèges accordés à la déesse de l'agriculture s'expliquent par les liens de la dynastie avec le culte de Déméter : Gélon était descendant de Télinès de Géla, premier hiérophante des deux déesses à l'origine du génos deinoménide des hiérophantes ${ }^{129}$. Ils s'expliquent aussi par l'omniprésence de la déesse en Sicile sur laquelle l'historien s'est longuement attardé dans son livre 5. Diodore ajoute que Gélon imposa aux Carthaginois la construction de deux temples que l'on identifie aujourd' hui comme ceux de la victoire à Himère, dans la ville basse, probablement consacré à Athéna, et celui d'Athéna à Syracuse. L'archéologie a en effet abondamment souligné le parallèle architectural entre les deux édifices et confirmé l'information diodoréenne qui les plaçait dans un même programme de construction ${ }^{130}$. Mais

I27 Athénée, 6.231 e-f (sur les offrandes de trépied à Delphes de la part des Deinoménides) et bien sûr Paus., 6.9. 4-5 (chars commémoratifs de Gélon à Olympie) ; 6.19.I4. (trésor de Géla à Olympie). La bibliographie est abondante sur le sujet. Cf. Jacquemin 1992 ; Rolley 1990 (sur l'aurige de Delphes, attribué au monument dédié par le fils de Hiéron ${ }^{\text {er }}$, Polyzèlos, pour commémorer les victoires pythiques de son père.

I28 Gras 1990.

I29 Hdt 7. 153.

I30 Mertens 1984.

DHA supplément 6 
l'attribution de ces deux temples à Gélon, que Diodore emprunte peut-être à Timée, a été battue en brèche par la typologie de certains fragments architecturaux : on a ainsi constaté que la métrologie correspondait à une norme agrigentine et non syracusaine, et que les protomés léonines qui couronnaient la sima de l'édifice avaient une parenté stylistique étroite avec celles du temple d'Héraklès à Agrigente. On a de ce fait proposé que la construction du temple de la Victoire ait été commanditée à la fois par Gélon et par Théron, ce qui serait assez conforme à l'équilibre politique voulu par les deux hommes et à la situation géopolitique de la région, sous domination agrigentine depuis une dizaine d'années ${ }^{131}$. On a souvent souligné le parti pris de Diodore, probablement influencé par la propagande deinoménide, qui exalte les qualités de Gélon au détriment de celles de Théron.

En revanche Diodore ne fait aucune référence à l'essor démographique et urbanistique des villes, pourtant indiqué par Hérodote qui mentionne le synœcisme des villes orientales, avec notamment la destruction de Mégara Hyblaea. Diodore a pu le traiter en digressions dans le livre $\mathrm{X}$ dont il ne reste que des fragments, mais c'est peu probable car il ne l'évoque pas non plus dans le bilan qu'il tire de la période gélonienne, à la mort du basileus, alors que les Syracusains lui rendent les honneurs réservés aux œcistes. Or l'essor de la ville, bien connu de l'archéologie syracusaine, date de cette époque. Un nouveau plan d'urbanisme est mis en place, donnant naissance à des quartiers qui déplacent le centre de gravité de la ville vers la colline de Temenites, où est construit dans le courant $\mathrm{du} \mathrm{V}^{\mathrm{e}}$ siècle, le premier théâtre, lieu de rassemblement de l'assemblée et édifice de spectacle, que la tradition attribuait à l'architecte Damocopos, dit Myrilla ${ }^{132}$. Là aussi on s'est interrogé sur la probable politique hydraulique des Deinoménides ${ }^{133}$, vu que sont connus à Syracuse quatre aqueducs et que Thucydide les évoque dans son livre 6. Les Athéniens coupent les canalisations qui portent l'eau en ville. Si le consensus s'accorde pour écarter de ces réalisations l'aqueduc du Galermi, la question reste posée pour les trois aqueducs du Nymphée, du Paradis et de Tremiglia qui présentent la même typologie architecturale.

L'Agyriote cite enfin quelques monuments funéraires prestigieux, comme les tombeaux de Théron à Agrigente ou de Gélon et de son épouse à Syracuse, que les archéologues ont parfois tenté d'identifier en se fondant sur la description diodoréenne. Â Agrigente, on a proposé des hypothèses plus ou moins fantaisistes pour le

I3I Mertens - Horn 1988.

132 Eudaimon Pelusii, dans Eustathe, Schol. Hom.Od. 3.68 : Diom. G.L. (Keil), I, p. 487 : textes cités par Troiani 198I ; Bernabò Brea 1967.

133 Collin Bouffier 1987 ; Guzzardi 2000 ; Wilson 2000. 
mausolée théronien depuis longtemps, en vain. L'archéologie, dans l'état actuel de la recherche, a seulement confirmé le soin donné à leurs sépultures par les Agrigentins. Ainsi la nécropole de Contrada Pezzino, l'une des plus importantes de la cité, a livré des monuments d'un type architectural somptueux et un mobilier de valeur, tout particulièrement à la fin du $\mathrm{VI}^{\mathrm{e}}$ et pendant tout le $\mathrm{V}^{\mathrm{e}}$ siècle où les importations de céramique attique prestigieuse montrent une hausse globale du niveau de vie des Agrigentins ${ }^{134}$. Ce n'est pas le cas à Syracuse, où la nécropole du Ve siècle, mal connue, n'a pas révélé de distinction particulière dans la typologie et le mobilier des tombes et ressemble à bon nombre de nécropoles contemporaines où le statut des individus est difficile à estimer à partir des vestiges funéraires. Là aussi, la localisation du tombeau de Gélon et de son épouse Damarétè a été l'objet d'hypothèses plus ou moins crédibles. Dans le livre II de sa Bibliothéque Historique (38), Diodore décrit les funérailles du tyran, soucieux de montrer la popularité de son règne :

« Les Syracusains avaient supprimé par une loi les obsèques (ekphora) somptueuses et réduit les dépenses accoutumées faites pour leurs défunts, mais ils avaient aussi pris l'habitude de négliger des prescriptions de la loi et en particulier ils négligeaient absolument les règles sur les funérailles (entaphia). Le roi Gélon, voulant favoriser le sens civique de son peuple dans tous les domaines, appliqua fermement à son propre égard la loi sur les funérailles (taphè). [...] il transmit la royauté à Hiéron, le plus âgé de ses frères et, relativement à ses funérailles (taphè), il lui donna des instructions précises, pour qu'il observât scrupuleusement la loi. Aussi, quand il fut mort son successeur dans la royauté suivit-il ses instructions dans la célébration de ses obsèques (ekphora). Son corps fut enterré dans la propriété de sa femme, dans les dites Neuf Tours, ouvrage dont le caractère imposant provoquait l'admiration. La foule entière des Syracusains lui fit cortège jusqu'à ce lieu, distant de deux cents stades (stadious diakosious). Après qu'il eut été enterré là, le peuple lui éleva un tombeau remarquable et lui décerna les honneurs héroïques ; plus tard les Carthaginois qui marchaient sur Syracuse renversèrent le monument et Agathocle jaloux en abattit les tours. $\gg^{135}$

Si la cérémonie répondait peut-être aux restrictions officielles, interprétées généralement dans le cadre général des lois somptuaires connues à la fin de l'époque archaïque et au début de l'époque classique ${ }^{136}$, il n'en est pas de même pour la sépulture. D'après une hypothèse de G. Voza ${ }^{137}$, ce monument funéraire serait situé sur la colline de Temenites, en amont du théâtre : ses fouilles y ont révélé une terrasse au centre de laquelle les fondations d'un édifice contenaient deux fosses à caisse monumentale et couverture plate de grande qualité. L'archéologue a proposé d'y voir les

\footnotetext{
I34 De Miro 1988, p. 248-252.

I35 Traduction Haillet remaniée.

136 Brugnone 1992, qui date cette loi du temps des Gamoroi.

I37 Voza 1993-94.
}

DHA supplément 6 
tombes de Gélon et de Damarétè. Bien que l'hypothèse fût séduisante, elle contredit le texte de Diodore qui situe la sépulture dans le territoire et la dote de neuf tours. On a relevé surtout l'exagération du chiffre donné par l'historien et de ce fait rejeté son témoignage : l'endroit serait éloigné de deux cents stades, soit 40km du centre urbain. Si l'on suppose une erreur de Diodore, le tombeau devait être situé plutôt dans un rayon de vingt stades, (eikosi, soit trois/quatre kilomètres), c'est-à-dire dans la plaine de l'Anapos, voire à l'extrémité occidentale ou septentrionale du plateau des Épipoles qui n'est pas enserré dans les fortifications avant le IV ${ }^{\mathrm{e}}$ siècle. Le tombeau, conformément à la tradition, est situé non seulement à l'extérieur du périmètre urbain, mais surtout dans la campagne, ce que n'est plus le quartier du théâtre à la mort du Deinoménide. Le terme d'agros employé ici par l'historien se rapporte toujours à un bien-fonds rural ${ }^{138}$ d'autant que les Carthaginois, en 396, n'ont pu avancer sur le territoire urbain désormais défendu par les fortifications dionysiennes et qu'à aucun moment les sources ne nous indiquent qu' ils ont pris position sur les Épipoles. On devrait alors rejeter également l'information de Diodore sur l'apparat de la sépulture : le texte utilise un terme peu diffusé pour mentionner la tour, tursis, et non pas purgos. Le mot tursis, qui a la même signification, est également utilisé pour désigner une maison fortifiée dans la campagne ${ }^{139}$. Le lexicographe Hésychius glose sur les différentes formes de cette racine, turris et tursos, dont il existerait un diminutif sicilien, turridion. S'agit-il d'un mausolée couronné de neuf tours ? le terme choisi suggérant une typologie spécifique ? Dans ce cas, il est difficilement envisageable au sommet d'un théâtre à moins de voir, là aussi, une exagération de l'historien et de réduire les tours à ces pyramides à degrés qui couronnent les tombes des élites à partir du renouveau timoléonien selon les dires mêmes de Diodore ${ }^{140}$. On connaît en effet, dans un certain nombre de nécropoles de Sicile, des stèles dites à épitymbia ${ }^{141}$, constituées d'une pyramide à degrés coiffant le

I38 On notera ici l'intérêt de la remarque : la propriété appartient à la femme de Gélon, ce qui suppose qu’à Syracuse, à l'époque archaïque et au début de l'époque classique, les femmes avaient le droit de propriété foncière.

I39 Chantraine, s.v. Tursis, qui cite comme exemple l'inscription d'Amorgos des $\mathrm{II}^{\mathrm{c}}-\mathrm{I}^{\mathrm{er}}$ siècles av. J.-C.

14016.83.

I4I Coarelli 1980. 
sommet de la tombe ${ }^{142}$ : elles se développent dans la seconde moitié du $\mathrm{IV}^{\mathrm{e}}$ siècle et à l'époque hellénistique, mais il est probable qu'il faille là aussi chercher un modèle antérieur.

Diodore nous offre donc une vision très positive, voire panégyrique de l'île au $\mathrm{V}^{\mathrm{e}}$ siècle, qui a tendance à gommer les vicissitudes d'une histoire événementielle somme toute assez troublée. Cette vision s'appuie sur une réalité géopolitique et économique qui résulte à la fois d'un développement amorcé dans les siècles précédents et des bouleversements du premier quart du $V^{\mathrm{e}}$ siècle, à la suite de l'accession au pouvoir de tyrans hors du commun et du conflit victorieux avec Carthage. Peu soucieux d'élaborer un jugement critique des types de régime qui se succèdent jusqu’à précipiter la Sicile dans « l'anarchie », comme la qualifient à Athènes les partisans de l'expédition de 4I5, et témoin de destructions et de répressions qu'il condamne lors de la guerre d'Octave en Sicile, Diodore préfère insister sur la prospérité et l'essor économique et culturel que connaît sa patrie au $V^{e}$ siècle av. J.-C. Son témoignage, même lacunaire, parfois partial, offre à l'archéologue de la Sicile des capacités de confrontation précieuses pour comprendre les réalités qu'il met au jour et saisir des modes de pensée et de faire propres à la société sicilienne du $\mathrm{V}^{\mathrm{e}}$ siècle.

\footnotetext{
I42 Pour le $V^{\mathrm{e}}$ siècle, on en connaît peu d'attestations : à Camarina, (Orsi 1904, col.790 ; fig. I2. L'archéologue s'étonne de l'absence quasi-totale d'épitymbia dans la nécropole de Passo Marinaro, absence qu'il attribue au statut inférieur de la population inhumée là). En revanche, ce type de marqueur funéraire est bien connu à partir du $\mathrm{IV}^{\mathrm{e}}$ siècle, pendant la période dite timoléonienne : à Léontinoi, dans la nécropole de la Valle San Mauro, cf. Rizza 1957, p. 71 ; Rizza 1955, en particulier, p. 339-340; à Butera, une nécropole d'une quarantaine de sépultures contemporaines dont douze à épitymbion (Adamesteanu 1958, col. 224-269); il se diffuse à l'époque hellénistique : voir en particulier à Centuripe (Libertini 1947, p. 304-307).
}

DHA supplément 6 


\section{Éditions de textes}

Casevitz 1972 : M. Casevitz, Diodore de Sicile. Bibliothèque Historique. Livre XII, Paris, CUF, 1972.

Barguet 1964 : A. Barguet, Hérodote : Enquête, Paris, La Pléiade, 1964.

Chamoux, Bertrac et Vernière 2002 : Fr. Chamoux, P. Bertrac et Y. Vernière, Diodore de Sicile. Bibliothéque Historique. Tome I. Introduction générale et livre I, Paris, CUF, 2002 [1993].

Haillet $200 \mathrm{I}$ : J. Haillet, Diodore de Sicile Bibliothèque Historique. Livre XI, Paris, CUF, $200 \mathrm{I}$.

\section{Bibliographie}

Adamesteanu 1958 : D.Adamesteanu, « Scavi e scoperte da 195 I al 1957 nella provincia di Caltanissetta » NSA, 1958, p. 288-382.

Alliata et Belvedere 1988 : V. Alliata, O. Belvedere et al., Himera 3, p. 379, Rome, 1988.

Ambaglio 1995 : D. Ambaglio, La Biblioteca storica di Diodoro Siculo: problemi e metodi, Côme, 1995.

Amouretti 1986 : M.-Cl. Amouretti, Le pain et l'huile dans la Grèce antique. De l'araire au moulin, Paris, I986.

Arnone 1991 : L. Arnone, Gl ipogei dell'Agrigentino, Agrigente, réédition [1952].

Asheri 1973 : D. Asheri, « La popolazione di Imera nel V sec.a.C », RFIC, IOI, 1973, p. 457-465.

Asheri 1992 : D. Asheri, « Agrigento libera: rivolgimenti interni e problemi costituzionali, ca 471-446.a.C., dans L.Braccesi et E. De Miro (éds.), Agrigento e la Sicilia greca. Atti della settimana di studio, Agrigento 2-8 maggio 1998, Rome, 1992, p. 95-1II.

Barbanera 1995 : M. Barbanera, Il guerriero di Agrigento, Rome, 1995.

Bejor 1975 : G. Bejor, « Ricerche di topografia e di archeologia romana nella Sicilia sud-occidentale », $A S N P$, 1975, V, 4, p. 1275-1303.

Bejor 1991 : G. Bejor, « Spunti diodorei e problematiche dell'archeologia siciliana », dans E. Galvagno et C. Molè Ventura (éds), Mito, storia, tradizione: Diodoro Siculo e la storiografia classica: atti del convegno internazionale Catania-Agira, 7-8 dicembre 1984, Catane, 1991. p. 255-269.

Belfiore 2000 : R. Belfiore, « Il centro abitato indigeno-ellenizzato di Piano dei Casazzi », SicArch. 98, 2000, p. 259-276.

Beltrame et Boetto 1997 : C. Beltrame and G. Boetto, « Macine da relitti », Archeologia Subacquea 2, 1997, p. 167-196.

Belvedere 200 I : O. Belvedere, « Il territorio di Himera e il problema della chora coloniale in Sicilia », dans Problemi della chora coloniale dall'Occidente al Mar Nero. Atti del Quarantesimo Convegno sulla Magna Grecia [Tarente, 29 septembre-3 octobre 2000], Tarente, 2001, p. 707-755.

Bernabò Brea 1967 : L. Bernabò Brea, « Studi sul teatro greco di Siracusa », Palladio, 17, p. 97-154.

Bianchi et al. 1998 : R. Bianchi, R.M. Cavalli, F. Colosi, M.C. Conti, C.M. Marino, S. Pignatti, S. Pino, M. Poscolieri, L. Versino et C. Zoppi, Selinunte IV, Rome, 1998. 
Biondi 2002 : G. Biondi, « Per una carta archeologica del territorio di Centuripe », dans G. Rizza (éd.), Scavi e ricerche a Centuripe, Catania, 2002, p. 4I-8I.

Blandini 1999 : G. Blandini, I templi di Agrigento, Segesta e Selinunte: storia, architettura, tecnica, Palerme, 1999.

Bove 1994 : A. Bove, « La tipologia strutturale dell'insediamento agricolo presente nella Sicilia centro-occidentale durant gli anni della dominazione romana, », SicArch., 84, 1994, p. 79-112.

Briant 1996 : P. Briant, Histoire de l'empire perse. De Cyrus à Alexandre, Paris, 1996.

Brugnone 1992 : A. Brugnone, « Le leggi suntuarie di Siracusa », PP, 1992, 47, p. 5-24.

BTCGI: G. Nenci et G.Vallet (éds), Bibliografia Topografica della colonizzazzione greca in Italia e nelle isole tirreniche, Pise-Rome, 1977].

Canzanella 1989: G. Canzanella, « Ficuzza », BTCGI, 7, 1989, p. 456.

Canzanella 1990 : G. Canzanella, « Gurgazzi », BTCGI, 8, 1990, p. 216-217.

Carter 2006 : J.C. Carter, Discovering the Greek countryside at Metaponto, Ann Arbor, 2006.

Casevitz I986 : M. Casevitz, « Quelques termes d'espace chez les comiques : kome, choros, chora et les dérivés », Ktèma, II, 1986, p. I29-136.

Castellana 1983: G. Castellana, « Nuove ricognizioni nel territorio di Palma di Montechiaro », $S A, 52 / 53$, 1983, p. I19-146.

Castellana 1990 : G. Castellana, « Granciforte », dans BTCGI 8, 1990, p. 169-170.

Chantraine 1999 : P. Chantraine et al., Dictionnaire étymologique de la langue grecque, Paris, éd. augmentée, 1999.

Coarelli 1980 : F. Coarelli, « La cultura figurativa in Sicilia nei secoli IV-III a.C. », dans E. Gabba et G. Vallet (éds), La Sicilia antica, Naples, 1980, II.I, p. 155-182.

Cohen-Skalli infra: A. Cohen-Skalli, « Le témoignage de Diodore de Sicile sur deux cités élymes : Ségeste et Éryx (VIc et V' siècles av. J.-C.) », dans S. Collin-Bouffier (éd.), Diodore d'Agyrion et l'histoire de la Sicile, infra, p. 137-153.

Collin Bouffier 1987 : S. Collin Bouffier, «L'alimentation en eau de la colonie grecque de Syracuse. Réflexions sur la cité grecque et sur son territoire», MEFRA, 99, 1987, 2, p. 661-69I.

Collin Bouffier 1999 : S. Collin Bouffier, «Les élites urbaines en Sicile grecque du VII ${ }^{\mathrm{e}}$ au Ve siècle av. J.-C. ou la reproduction d'un modèle homérique», dans Cl. Petitfrère (éd.), Construction, reproduction et représentation des patriciats urbains de l'Antiquité au XX $X^{e}$ siècle, Colloque du CEHVI, Tours, [7-9 septembre 1998], Tours, 1999, p. 363-373.

Collin Bouffier 2000 : S. Collin Bouffier, «Quelles fonctions pour la kolymbethra d'Agrigente ? », dans G.C.M. Jansen (éd.), Cura aquarum in Sicilia: proceedings of the tenth International Congress on the History of Water Management and Hydraulic Engineering in the Mediterranean Region, Syracuse, May 16-22, 1998, BABESCH, supplément 6, Leyde, 2000, p. 37-43.

Collin Bouffier 2001 : S. Collin Bouffier, « Les adductions hydrauliques des villes grecques de Sicile : des ouvrages techniques méconnus», dans J.-P. Brun et Ph. Jockey (éds), TEXNAI. Techniques et sociétés en Méditerranée, Paris, 2001, p. 513-528. 
Collin Bouffier 2002 : S. Collin Bouffier, « Eau et campagne en Sicile grecque : observations préliminaires à l'étude », dans IN BINOS ACTUS LUMINA. Rivista di studi e ricerche sull' idraulica storica e la storia della tecnica, Atti del Convegno Internazionale di Studi su Metodologie per lo studio della scienza idraulica antica. Ravenna, I3-15 mai 1999, La Spezia, 2002, p. 27-35.

Collin Bouffier 2008 : S .Collin Bouffier, « La gestion de l'eau dans les territoires grecs », dans E. Hermon (dir.), Vers une gestion intégrée de l'eau dans l'empire romain, colloque de l'université Laval (Québec), [2006], Rome, 2008, p. 4I-52.

Collin Bouffier 2009 : S. Collin Bouffier, « L'eau en Sicile grecque. Nouvelles perspectives de recherches », dans S. Péré-Noguès (éd.), L'état de la recherche sur la Sicile antique, Journée d'étude Toulouse, 25 octobre 2007], Pallas, 79, 2009, p. 65-79.

Collin Bouffier 2010 : S. Collin Bouffier, « Les élites face au tyran en Sicile grecque à l'époque classique », dans L. Capdetrey et Y. Lafond (éds), Les cités et ses élites. Pratiques et représentations des formes de domination et de contrôle social dans les cités grecques, Bordeaux, 2010, p. 291-305.

Cusumano, infra: N. Cusumano, « Gérer la haine, fabriquer l'ennemi. Grecs et Carthaginois en Sicile entre les V' et IV siècles av. J.-C. », dans S. Collin-Bouffier (éd.), Diodore d'Agyrion et l'histoire de la Sicile, infra, p. 113-135.

De Angelis 2000 : Fr. De Angelis, « Estimating the Agricultural Base of Greek Sicily », PBSR, 68, 2000, p. III-I 48 .

De Angelis 2000-200I : Fr. De Angelis, « Archaeology in Sicily 1996-2000 », AR, 47, 2000 - 2001, p. 145 -20I.

De Angelis 2006a : Fr. De Angelis, « Archaeology in Sicily 200I-2005 », AR, 53, 2006, p. 123-190.

De Angelis 2006b : Franco De Angelis, « Going against the grain in Sicilian Greek economics », G $\mho R$, 2006, Ser. 2 53.1, p. 29-47.

De Miro 1988 : E. De Miro, « Akragas, città e necropoli nei recenti scavi », dans Veder greco ad Agrigento, Rome, 1988, p. 235-252.

De Miro 2000 : E. De Miro (éd.), Agrigento. I santuari urbani: I'area sacra tra il Tempio di Zeus e Porta V, Rome, 2000.

Devillers 1998 : Olivier Devillers, «Un portrait « césarien » de Gélon chez Diodore de Sicile (XI, 20-26) », dans $A C$, 1998, 67, p. 149-167.

De Waele 1980 : J.A. De Waele, « La popolazione di Akragas antica », dans Miscellanea di studi classici in onore di Eugenio Manni, 3, Rome, 1980, p. 747-760.

De Waele 1982 : J.A. De Waele, «I frontoni dell'Olympion agrigentino », dans Aparchai. Nuove ricerche e studi sulla Magna Grecia e la Sicilia antica in onore di Paolo Enrico Arias, Pise, 1982, p. 27 I-278.

De Waele 1989 : J.A. De Waele, « Die Entwurf der dorischen Tempel von Akragas », AA, 1989, p. 180-24I.

Di Bella et Santagati 1998 : V. Di Bella et F.Santagati, « Prospezione archeologica nel territorio costiero tra Agrigento e Siculiana $\gg$, SicArch 96, 1998, p. 71-87.

Di Stefano C.A. 2008 : C. A. Di Stefano (éd.), Demetra. la divinità, i santuari, il culto, la leggenda : atti del I congresso internazionale, Enna, I-4 luglio 2004, Pise-Rome, 2008. 
Di Stefano G. 200 I : G. Di Stefano, « La chôra di Camarina », dans Problemi della chora coloniale dall'Occidente al Mar Nero. Atti del Quarantesimo Convegno sulla Magna Grecia [Tarente, 29 septembre-3 octobre 2000], Tarente, 200I, p. 689-705.

Di Stefano et Anelli 200 I : G. Di Stefano et M. Anelli, « La 'Fattoria delle api' sull'Irminio. La ceramica d'uso comune », $S A$, 99, 200I, p. 31-50.

Di Vita et Costantini 1983: A. Di Vita et L. Costantini, « Camarina 1958. Documenti e note », $B A$, 1983 LXVIII N ${ }^{\circ} 17$, p. 3I-44.

Dubois 1989 : L. Dubois, Inscriptions grecques dialectales de Sicile : contribution à l'étude du vocabulaire grec colonial, Rome, 1989.

Entella $200 \mathrm{I}$ : Da un'antica città di Sicilia: i decreti di Entella e Nakone: catalogo della mostra, Pise, $200 \mathrm{I}$.

Fantasia 1993: U. Fantasia, « Grano siciliano in Grecia nel V e IV secolo », ASNP, s.3, 23, 1993, p. 9-31.

Frasca 1997 : M. Frasca, « È anonima la città siculo-greca di Monte San Mauro di Caltagirone? », PP, 52, 1997, p. 407-17.

Gallo 1989 : L. Gallo, « Produzione cerealicola e demografia siciliana », AION, II, 1989, p. 3 I-53.

Gallo 1992 : L. Gallo, « La Sicilia occidentale e l'approvvigionamento cerealicolo di Roma », ASNP, 22, 1992, p. 365-398.

Gallo 200I : L. Gallo, « Le fonti letterarie sulle chorai coloniali », dans Problemi della chora coloniale dall'Occidente al Mar Nero. Atti del Quarantesimo Convegno sulla Magna Grecia [Tarente, 29 septembre-3 octobre 2000], Tarente, 200I, p. 49-7I.

Garnsey 1996 : P. Garnsey, Famine et approvisionnement dans le monde gréco-romain, Paris, 1996 [1988].

Giudice et al. 2003 ; F. Giudice et al., « Il percorso della mostra. Le importazioni attiche a Gela: costruzione del quadro di riferimento », dans R. Panvini et F. Giudice (éds), TA ATTIKA. Veder greco a Gela. Ceramiche attiche figurate dall'antica colonia, [Géla-Syracuse-Rhodes], Rome, 2003, p. 23-92.

Giustolisi 1981 : V. Giustolisi, Camico, Triocala, Caltabellotta, Palerme, 198I.

Gras 1990: M. Gras, « Gélon et les temples de Sicile après la bataille d'Himère », AION, I2, 1990, p. 59-68.

Greco 1999a : E. Greco, « Siculi ed Enotri: tra analogie e differenze » dans La colonisation grecque en Méditerranée occidentale: Actes de la rencontre scientifique en hommage à Georges Vallet, [Naples, 15-18 novembre 1995], Rome, 1999, p. 281-292.

Greco 1999b : E. Greco (éd.), La città greca antica. Istituzioni, società, forme urbane, Rome, 1999.

Greco 2001 : E. Greco, « Abitare in campagna », dans Problemi della chora coloniale dall'Occidente al Mar Nero. Atti del Quarantesimo Convegno sulla Magna Grecia [Tarente, 29 septembre-3 octobre 2000], Tarente, 200I, p. 17I-20I.

Guzzardi 2000 : L. Guzzardi, «Il complesso di viale Scala Greca e l'acquedotto del Paradiso a Siracusa », dans G.C.M. Jansen (éd.), Cura aquarum in Sicilia: proceedings of the tenth International Congress on the History of Water Management and Hydraulic Engineering in the Mediterranean Region, Syracuse, May 16-22, 1998, BABESCH, supplément 6, Leyde, 2000, p. 97-I0I. 
Guzzardi 2001 : L. Guzzardi, « Il territorio di Noto nel periodo greco », dans F. Balsamo et V. La Rosa (éds), Contributi alla geografia storica dell'agro netino. Atti delle giornate di studio, Noto, Palazzo Trugona, 29-30-3I maggio 1998, Noto, 2001, p. 97-109.

Hansen 2004 : M.H. Hansen, « The Concept of the Consumption City applied to the Greek Polis », dans T.H. Nielsen (éd.), Once Again: Studies in the Ancient Greek Polis, Stuttgart, 2004, p. 9-47.

Hansen 2006a : M.H. Hansen, The shotgun Method. The demography of the ancient Greek-City State Culture, Columbia et Londres, 2006.

Hansen 2006b : M.H. Hansen, Studies in the population of Aigina, Athens and Eretria, Copenhague, 2006.

Hansen et Nielsen 2004 : M.H. Hansen et T.H. Nielsen, An Inventory of Archaic and Classical Poleis, Oxford, 2004.

Hellmann 1999 : M.Chr. Hellmann, Choix d'inscriptions architecturales grecques, Lyon, 1999.

Hinz 1998 : V. Hinz, Der Kult von Demeter und Kore auf Sizilien und Magna Graecia, Wiesbaden, 1998.

Insediamenti rurali nella Sicilia antica. Atti delle Giornate di studio su gli insediamenti rurali nella Sicilia antica (1992; Caltagirone), Aitna, Quaderni di topografia antica, Catane, 1996.

Jacquemin 1992 : A. Jacquemin, « Offrandes monumentales italiotes et sikéliotes à Delphes », dans La Magna Grecia e igrandi santuari della madrepatria. Atti del trentunesimo convegno di studi sulla Magna Grecia, [1991], Tarente, 1992, p. 193-204.

La Genière 1999 : J. de La Genière, « De la céramique pour les mercenaires », dans La colonisation grecque en Méditerranée occidentale: Actes de la rencontre scientifique en hommage à Georges Vallet, [Naples, I5-18 novembre 1995], Rome, 1999, p. 121-130.

La Genière 2003: J. de La Genière, « Vasi attici dalle necropoli di Gela », dans R. Panvini et F. Giudice (éds), TA ATTIKA. Veder greco a Gela. Ceramiche attiche figurate dall'antica colonia, [Géla-Syracuse-Rhodes], Rome, 2003, p. 149-155.

Lamagna 1993-94: G. Lamagna, « Le ultime ricerche archeologiche nei territori di Adrano e Caltagirone », Kokalos, 39-40,1993-94, p. 873-879.

Laudani, Collin Bouffier et al. s. p. : S. Laudani, S. Collin Bouffier, J. France, L. Arcifa, V. Vigiano, N. Blando, «Entrepôts céréaliers en Sicile de l'Antiquité à l'époque moderne », dans C. Virlouvet et B. Marin (éds), Le ravitaillement des cités de la Méditerranée : entrepôts et trafics annonaires- Antiquité -Temps Modernes, Aix-en-Provence-Le Caire-Rome, s. p.

Libertini 1947 : G. Libertini, « Centuripe. Scavi nella necropoli in contrada Casino, NSA, 1947, p. 259-311. Luraghi 1994 : N. Luraghi, Tirannidi arcaiche della Sicilia et della Magna Grecia, Florence, 1994.

Mc Connell 1989 : B. E. Mc Connell, « Gaddimi », dans BTCGI, 7, 1989, p. 528-29.

Mc Connell 1990 : B. E. Mc Connell, « Guardabasso », dans BTCGI, 8, 1990 p. 214-215.

Mc Dougall 1983 : J. Lain Mc Dougall, Lexicon in Diodorum Siculum, Hildesheim - Zürich - New York, 1983.

Maffoda 1996 : G. Mafodda, La monarchia di Gelone tra pragmatismo, ideologia e propaganda, Messina, 1996. 
Malone, Ayala et al. 200I-2003: C. Malone, G. Ayala, M. Fitzjohn and S. Stoddart, « Under the Volcano », Accordia Research Papers 9, 200I-2003, p. 7-21.

Marconi 1997 : C. Marconi, «I Titani e Zeus Olimpio. Sugli Atlanti dell'Olympieion di Agrigento », Prospettiva, 87-88, 1997, p. 2-13.

Martin 1987 : R. Martin, « Aspects financiers et sociaux des programmes de construction dans les villes grecques de Grande Grèce et de Sicile » [1973], dans R. Martin, Architecture et urbanisme, Athènes -Rome, 1987.

Meister 1967 : K. Meister, Die sizilische Geschichte bei Diodor von den Anfangen bis zu den Tod Agathokles, Quellenuntersuchungen zu Buch IV-XXI, Diss. Munich, 1967.

Mertens 1984 : D. Mertens, Der Tempel von Segesta und die dorische Tempelbaukunst des griechischen Westens in klassischer Zeit, Mayence, 1984.

Mertens 2006 : D. Mertens, Città e monumenti dei Greci d'Occidente, Rome, 2006.

Mertens-Horn 1988 : M. Mertens-Horn, Die LöwenkopfWasserspeier des griechischen Westens im 6. und s. Jahrbundert v.Chr., RM 9g, Mayence, 1988.

Messina 2009 : H. Messina, « Lo scavo in Via Zappalà (Siracusa) », Kokalos, XLII-XLVIII, t.2, 2009, p. $807-819$.

Miccichè 1989 : C. Miccichè, Mesogheia. Archeologia e storia della Sicilia centro-meridionale dal VII al IV secolo a.C., Caltanissetta, 1989 .

Miccichè 1996 : C. Miccichè, Gli Ipogei agrigentini tra archeologia, storia e mitologia, Agrigente, 1996.

Morris 1987 : I. Morris, Burial and Ancient Society: the Rise of the Greek City, Cambridge/ New York, 1987.

Morris et Tusa 2004 : I. Morris et S. Tusa, « Scavi sull'acropoli di Monte Polizzo, 2000-2003 », SA, 102, 2004 , p. 35-90.

Muggia 1997 : A. Muggia, L'area di rispetto nelle colonie magno-greche e siceliote, Palerme, 1997.

Nenci 1993 : G. Nenci, « Agrigento e la Sicilia greca nel quadro dei rifornimenti granari del mondo greco », $A S N P$, s.3, 23, 1993, p. I-8.

Orsi 1904 : P. Orsi, « Camarina. Campagne archeologiche del I899 e I903 », MonAL, I4, 1904, col.757-955.

Panvini 1993-94 : R. Panvini, 'Ricerche nel territorio di Monte San Giuliano (CL), Monte Desusino, S. Giovanni Gemini, Caltabellotta, Sant'Anna », Kokalos, 39-40,1993-94, p. 755-770.

Pearson 1987 : L. Pearson, The Greek Historians of the West. Timaeus and His Predecessors, Atlanta, 1987.

Péré-Noguès infra : S. Péré-Noguès, « Diodore de Sicile et les Sikèles : histoire et/ou mémoire d'un «ethnos » et de son « héros » Doukétios », dans S. Collin-Bouffier, Diodore d'Agyrion et l'histoire de la Sicile, DHA, p. 155-170.

Prestianni Giallombardo 1998 : A.M. Prestianni Giallombardo (éd.), Colloquio Alesino. Atti del colloquio tenutosi il 7 maggio Ig9s in S. Maria delle Palate (Tusa), Catania, 1998.

Procelli et Albanese 1992 : E. Procelli et R.M. Albanese, « Ramacca. Saggi di scavo nelle contrade Castellito e Montagna negli anni 1978, 1981 et 1982 », NSA 1988-89, suppl.I, 1992, p. 7-150.

Procelli 1989, Aspetti e problemi dell'ellenizzazione calcidese nella Sicilia orientale », MEFRA, 101.2, 1989, p. 679-689.

DHA supplément 6 
Rizza 1955 : G. Rizza, « Leontini. Campagne di scavi 1950-1951 e I95I-1952; la necropoli di Valle S.Mauro; le fortificazioni meridionali della città e la porta di Siracusa $\gg, N S A$, 1955, p. 281-376.

Rizza 1957 : G. Rizza, «Leontini. Scavi e ricerche degli anni 1954-1955 », BA, 42, 1957, p. 63-73.

Rolley 1990 : Cl. Rolley, « En regardant l'aurige », BCH, II 4, 1990, p. 285-297.

Sacks $1990:$ K. Sacks, Diodorus and the first century, Princeton, 1990.

Sacks 1994 : K.S. Sacks, « Diodurus and his sources: Conformity and Creativity », dans S. Hornblower (éd.), Greek Historiography, Oxford, 1994, p. 213-232

Siracusano 1983: A. Siracusano, Il santuario rupestre di Agrigento in località San Biagio, Rome, 1983.

Sjöqvist 1962 : E. Sjöqvist, «I Greci a Morgantina », Kokalos, 7, 1962, p. 52-68.

Spatafora 1993 : Fr. Spatafora, « Un gruppo di macine da Monte Castellazzo di Poggioreale », dans Studi sulla Sicilia Occidentale in onore di Vincenzo Tusa, Padoue, 1993, p. 165- 171.

Spigo 1980-8I : U. Spigo, « Ricerche a Monte San Mauro, Francavilla di Sicilia, Acireale, Adrano, Lentini, Solarino », Kokalos, 26-27, 1980-81, 2.I, p. 771-795.

Spigo 1986 : U. Spigo, « L'anonimo centro greco di Monte San Mauro di Caltagirone nel quadro del arcaismo siceliota: prospettive di ricerca $\gg, M G R$, Rome, 1986, p. I-32.

Tagliamonte 1994 : G. Tagliamonte, I figli di Marte: mobilità, mercenarii e mercenario italici in Magna Grecia e Sicilia, Rome, 1994.

Thompson $200 \mathrm{o}$ : S.M. Thompson, « Problemi e principi di metodologia della ricognizione archeologica, dans Problemi della chora coloniale dall'Occidente al Mar Nero. Atti del Quarantesimo Convegno sulla Magna Grecia [Tarente, 29 septembre-3 octobre 2000], Tarente, 2001, p. 403-422.

Thompson 2002 : S. M. Thompson, dans P. Attema et al. (éds), New Developments in Italian Landscape Archaeology (BAR International Series), vol. 1091, Oxford, 2002, p. 76-82.

Troiani 1981 : M. Troiani, « Prima parte », dans L. Polacco et C. Anti, Il teatro antico di Siracusa, I, Rimini, I98I.

Turco 1990 : M. Turco, « Cassibile. Appunti per una carta archeologica del territorio », $S A, 72$, 1990, p. $67-78$.

Vallet 1962 : G. Vallet, « La colonisation chalcidienne et l'hellénisation de la Sicile orientale, Kokalos, 8, 1962, p. 31-47.

Van Compernolle R. 1992 : R. Van Compernolle, « La signoria di Terone », dans L. Braccesi et E. De Miro (éds.), Agrigento e la Sicilia greca. Atti della settimana di studio, Agrigento 2-8 maggio 19g8, Rome, 1992, p. 6I-75.

Van Compernolle Th. 1992 : Th. Van Compernolle, L'influence de la politique des Deinoménides et des Emménides sur l'architecture et l'urbanisme sicéliotes, Louvain, 1992.

Veder greco 1988 : Veder greco ad Agrigento, Rome, 1988.

Voza 1993-94 : G. Voza, « Attività della Soprintendenza di Siracusa e Ragusa », Kokalos, XXXIX-XL, 1993-94, p. I28I-I294.

Wilson 1980 : R. J. A. Wilson et A. Leonard Jr, « Field Survey at Heraclea Minoa (Agrigento) », JFA, Vol. 7, No. 2 (Summer 1980), p. 219-239. 
Wilson 1990 : R. J. A. Wilson, Sicily under the Roman Empire. The Archaeology of a Roman Province 36 $B C-A D$ 535, Warminster, 1990.

Wilson 1994 : R. J. A. Wilson, « Rural Settlement in Hellenistic and Roman Sicily: Excavations at Campanaio (AG), 1994-8 », Papers of the British School at Rome, Vol. 68 (2000), p. 337-369.

Wilson 1995 : R. J. A. Wilson, « Archaeology in Sicily 1988-1995 », AR, 42, 1995-1996, p. 59-123.

Wilson 2000 : R. J. A. Wilson, « Aqueducts and Water Supply in Greek and Roman Sicily: the present status quaestionis », dans G.C.M. Jansen (éd.), Cura aquarum in Sicilia: proceedings of the tenth International Congress on the History of Water Management and Hydraulic Engineering in the Mediterranean Region, Syracuse, May 16-22, 1998, BABESCH, supplément 6, Leyde, 2000, p. 5-36.

Zoppi 1999 : C. Zoppi, « Finito e non finito il tempio di Vulcano ad Agrigento », dans Studi di archeologia classica dedicati a Giorgio Gullini per i quarant'anni d'insegnamento, Alessandria, p. 211-20. 